\title{
Uniqueness of Lagrangian self-expanders
}

\author{
JASON D LOTAY \\ ANDRÉ NEVES
}

\begin{abstract}
We show that zero-Maslov class Lagrangian self-expanders in $\mathbb{C}^{n}$ that are asymptotic to a pair of planes intersecting transversely are locally unique if $n>2$ and unique if $n=2$.
\end{abstract}

53D12; 53C44

\section{Introduction}

Self-similar solutions to mean curvature flow model the flow behaviour near a singularity. If the initial condition for the flow is a zero-Maslov class Lagrangian in $\mathbb{C}^{n}$, it is well known (Neves [17, Corollary 3.5]) that self-shrinkers are trivial (ie, stationary solutions) and so the ones left to study are self-expanders. These are Lagrangians $L \subset \mathbb{C}^{n}$ so that $L_{t}=\sqrt{2 t} L$ is a solution to mean curvature flow.

Moreover, it is shown in Neves and Tian [19] that blow-downs of eternal solutions to Lagrangian mean curvature flow (like translating solutions for instance) are selfexpanders for positive time. Thus if one wants to understand whether or not non-trivial translating solutions can occur as blow-ups of finite time singularities of Lagrangian mean curvature flow, it is important that we understand self-expanders first.

Another related perspective on self-expanders is that they are the simplest solutions to mean curvature flow that start on cones and hence could be seen as models for starting the flow with singular initial condition.

The first examples of Lagrangian self-expanders were constructed in Anciaux [1] and Lee and Wang [13; 14]. In [10], Joyce, Lee and Tsui generalized these constructions and in particular they found, for any two Lagrangian planes $P_{1}, P_{2} \subset \mathbb{C}^{n}$ satisfying an angle criterion, explicit examples of zero-Maslov class Lagrangians asymptotic to these planes. They are diffeomorphic to $S^{n-1} \times \mathbb{R}$ and can be seen as the equivalent of Lawlor necks for the self-expander equation. The construction in [10] is quite general and provides examples that are asymptotic to non-stationary cones and examples that have Maslov class. Further examples were constructed in Castro and Lerma [3]. 
Given a Lagrangian cone in $\mathbb{C}^{n}$ that is graphical over a real plane and such that the Hessian of the potential function has eigenvalues uniformly in $(-1,1)$, Chau, Chen and $\mathrm{He}$ [4] showed there is a unique graphical Lagrangian self-expander asymptotic to that cone.

Let $P_{1}, P_{2} \subset \mathbb{C}^{n}$ be two Lagrangian planes intersecting transversely, denote the space of bounded smooth functions with compact support by $C_{0}^{\infty}\left(\mathbb{C}^{n}\right)$ and let $\mathcal{H}^{n}$ be $n-$ dimensional Hausdorff measure. We let $P_{1}+P_{2}$ denote the varifold whose support is given by the union of the planes $P_{1} \cup P_{2}$.

Definition 1.1 We say the self-expander $L$ is asymptotic to $L_{0}=P_{1}+P_{2}$ if

$$
\lim _{t \rightarrow 0} \int_{\sqrt{2 t} L} \phi \mathrm{d} \mathcal{H}^{n}=\int_{L_{0}} \phi \mathrm{d} \mathcal{H}^{n}
$$

for all $\phi \in C_{0}^{\infty}\left(\mathbb{C}^{n}\right)$.

In this paper we first show local uniqueness.

Theorem A Assume that neither $P_{1}+P_{2}$ nor $P_{1}-P_{2}$ are area-minimizing.

Let $L$ be a smooth zero-Maslov class Lagrangian self-expander in $\mathbb{C}^{n}$ asymptotic to $P_{1}+P_{2}$.

There exist $R_{0}>0$ and $\varepsilon>0$ so that any smooth zero-Maslov class Lagrangian self-expander that is

- asymptotic to $P_{1}+P_{2}$,

- $\varepsilon$-close in $C^{2}$ to $L$ in $B_{R_{0}}$,

coincides with $L$.

The idea for the proof of Theorem A is classical. We show that the linearization of the self-expander equation defines a Banach space isomorphism and then we apply the Inverse Function Theorem to obtain local uniqueness.

When $n=2$ we improve this result and show global uniqueness.

Theorem $\mathbf{B}$ Assume that neither $P_{1}+P_{2}$ nor $P_{1}-P_{2}$ are area-minimizing.

Smooth zero-Maslov class Lagrangian self-expanders asymptotic to $P_{1}+P_{2}$ are unique and thus coincide with one of the examples found by Joyce, Lee and Tsui. 
Remark 1.2 It is known that special Lagrangians in $\mathbb{C}^{2}$ that are asymptotic to a pair of planes are unique modulo scaling and rigid motions. This uses the fact that, after a hyperkähler rotation of the complex structure, special Lagrangian surfaces become holomorphic curves. No similar characterization holds for Lagrangian self-expanders, and hence the need for a different idea in Theorem B.

Moreover, without the smoothness assumption the uniqueness statement does not hold, as can be seen in Nakahara [15].

We now briefly describe the idea behind the proof of Theorem B.

The key result is to show Theorem 6.1, which says that the set of self-expanders in $\mathbb{C}^{2}$ that are asymptotic to a pair of transverse planes is compact. Assuming this result, the idea, given a pair of planes $P_{1}, P_{2}$ as in Theorem $\mathrm{B}$, is to deform $P_{2}$ into a plane $Q_{2}$ so that the Lagrangian angle remains constant and $P_{1}, Q_{2}$ become equivariant, ie, share the same $S^{1}$-symmetry. From Theorem A we can accompany the deformation of the planes with a (local) deformation of any self-expander $L$ asymptotic to $P_{1}+P_{2}$. Theorem 6.1 ensures that this local deformation can be carried all the way until we obtain a self-expander $Q$ asymptotic to $P_{1}+Q_{2}$. Since $P_{1}, Q_{2}$ are equivariant, it is simple to show that $Q$ is unique (see Lemma 7.1) and hence $L$ must have been unique as well.

Roughly speaking, the proof of Theorem 6.1 rests on the fact that every non-trivial special Lagrangian cone in $\mathbb{C}^{2}$ has area-ratios not smaller than two, but the area-ratios of any self-expander as in Theorem B are strictly smaller than two, ie, the area-ratios are too small for a singularity to develop.

Organization In Section 2 we introduce the basic concepts.

In Section 3 we show that zero-Maslov class self-expanders asymptotic to a transverse intersection of planes have exponential decay outside a compact set.

In Section 4 we develop the Fredholm theory for the linearization of the self-expander equation.

In Section 5 we show that zero-Maslov class self-expanders in $\mathbb{C}^{n}$ that are asymptotic to a transverse intersection of a non-area-minimizing pair of planes are locally unique. This implies Theorem A.

In Section 6 we show that, given a compact set of transversely intersecting non-areaminimizing pairs of planes in $\mathbb{C}^{2}$, the family of zero-Maslov class self-expanders in $\mathbb{C}^{2}$ asymptotic to those pairs of planes is also compact. 
In Section 7 we use the work of the previous section and Section 4 to show global uniqueness for zero-Maslov class self-expanders in $\mathbb{C}^{2}$ that are asymptotic to a transverse intersection of a non-area-minimizing pair of planes. This proves Theorem B.

\section{Acknowledgements}

Both authors would like to thank Dominic Joyce for comments on an earlier version of this paper and the referee for the extensive comments that helped improve the exposition of the paper.

The first author was supported by an EPSRC Career Acceleration Fellowship. The second author was partly supported by Marie Curie IRG Grant and ERC Start Grant.

\section{Basic theory and notation}

Consider $\mathbb{C}^{n}$ with its usual complex coordinates $z_{j}=x_{j}+i y_{j}$, for $j=1, \ldots, n$, complex structure $J$, Kähler form $\omega=\sum_{j=1}^{n} \mathrm{~d} x_{j} \wedge \mathrm{d} y_{j}$ and holomorphic volume form $\Omega=\mathrm{d} z_{1} \wedge \cdots \wedge \mathrm{d} z_{n}$. Observe that the Liouville form $\lambda=\sum_{j=1}^{n}\left(x_{j} \mathrm{~d} y_{j}-y_{j} \mathrm{~d} x_{j}\right)$ satisfies $\mathrm{d} \lambda=2 \omega$.

Let $L$ be a connected Lagrangian in $\mathbb{C}^{n}$; that is, $L$ is a (real) $n$-dimensional submanifold of $\mathbb{C}^{n}$ such that $\left.\omega\right|_{L} \equiv 0$. Let $\boldsymbol{x}$ denote the position vector on $L$, let $\nabla$ be the (induced) Levi-Civita connection on $L$ and let $H$ be the mean curvature vector on $L$. We adopt the convention that $H$ is the trace of the second fundamental form on $L$. Standard Euclidean differentiation is denoted by $\bar{\nabla}$.

Notice that $\lambda$ is trivially a closed 1 -form on $L$. We say that $L$ is exact if there exists $\beta \in C^{\infty}(L)$ such that $\mathrm{d} \beta=\left.\lambda\right|_{L}$.

Since $\left.\Omega\right|_{L}$ is a unit complex multiple of the volume form at each point on $L$, we may define the Lagrangian angle $\theta$ on $L$ by the formula $\left.\Omega\right|_{L}=e^{i \theta} \operatorname{vol}_{L}$. We also have the relation $H=J \nabla \theta$ (cf Thomas and Yau [22, Lemma 2.1]). The Maslov class on $L$ is defined by the cohomology class of $\mathrm{d} \theta$, so $L$ has zero-Maslov class if $\theta$ is a single-valued function.

Observe that, since $\left.T \mathbb{C}^{n}\right|_{L}=T L \oplus N L$, we may decompose any vector $\boldsymbol{v}$ on $L$ into tangential and normal components, denoted $v^{\top}$ and $v^{\perp}$ respectively.

Definition 2.1 We say that $L$ is a self-expander if $H=\kappa \boldsymbol{x}^{\perp}$ for some $\kappa>0$. By rescaling $L$ we may assume that $\kappa=1$. 
The importance of self-expanders $L$ with $H=x^{\perp}$ is that $L_{t}=\sqrt{2 t} L$ for $t>0$ solves mean curvature flow.

We have the following basic properties of self-expanders.

\section{Lemma 2.2}

(i) Lagrangian self-expanders with zero-Maslov class are exact.

(ii) Let $L$ be a zero-Maslov class self-expander. Then $L$ is a self-expander with $H=\boldsymbol{x}^{\perp}$ if and only if $\beta+\theta$ is constant.

Proof Let $L$ satisfy $H=\boldsymbol{x}^{\perp}$. Since $H=J \nabla \theta$, one sees that

$$
\nabla \theta=-J\left(\boldsymbol{x}^{\perp}\right)=-(J \boldsymbol{x})^{\top},
$$

so $\left.\lambda\right|_{L}=-\mathrm{d} \theta$. This proves the first property.

To prove the second property note that

$$
H-\boldsymbol{x}^{\perp}=0 \Longleftrightarrow \nabla \theta+J \boldsymbol{x}^{\perp}=0 \Longleftrightarrow \nabla \theta+(J \boldsymbol{x})^{\top}=0 \Longleftrightarrow \nabla(\theta+\beta)=0 .
$$

Let $P_{1}, P_{2}$ be two Lagrangian planes intersecting transversely. From Ilmanen [7, Section 7.2], there exists a constant $C_{0}$ such that whenever a self-expander $L$ is asymptotic to $P_{1}+P_{2}$ then

$$
\mathcal{H}^{n}\left(L \cap B_{R}\right) \leq C_{0} R^{n} \text { for all } R>0,
$$

where $B_{R}$ will always denote $B_{R}(0)$, the ball of radius $R$ about 0 in $\mathbb{C}^{n}$.

In this paper, all self-expanders $L$ we consider have the following properties:

- $L$ is Lagrangian with zero-Maslov class.

- $L$ has $H=\mathbf{x}^{\perp}$.

- $L$ is asymptotic to $L_{0}=P_{1}+P_{2}$, where $P_{1}, P_{2}$ are transversely intersecting Lagrangian planes.

We abuse notation and often identity the varifold $L_{0}=P_{1}+P_{2}$ with its support $L_{0}=P_{1} \cup P_{2}$.

A key tool in studying self-expanders is the backwards heat kernel.

Definition 2.3 Given any $\left(x_{0}, l\right)$ in $\mathbb{C}^{n} \times \mathbb{R}$, we consider the backwards heat kernel

$$
\Phi\left(x_{0}, l\right)(x, t)=\frac{\exp \left(-\frac{\left|x-x_{0}\right|^{2}}{4(l-t)}\right)}{(4 \pi(l-t))^{n / 2}} .
$$


Given a solution $\left(L_{t}\right)_{t>0}$ to mean curvature flow and $x_{0} \in \mathbb{C}^{n}, l>0$, we consider

$$
\Theta_{t}\left(x_{0}, l\right)=\int_{L_{t}} \Phi\left(x_{0}, l\right)(x, 0) \mathrm{d} \mathcal{H}^{n} .
$$

Note that when $L_{t}=\sqrt{2 t} L$, where $L$ is a self-expander, we have that $\Theta_{t}\left(x_{0}, l\right)$ is finite for $0<t<l$ due to (1) (see Ecker [5, Lemma C.3]).

Definition 1.1 implies that for all $x_{0} \in \mathbb{C}^{n}$ and $l>0$

$$
\lim _{t \rightarrow 0} \Theta_{t}\left(x_{0}, l\right)=\int_{L_{0}} \Phi\left(x_{0}, l\right) \mathrm{d} \mathcal{H}^{n}=\Theta_{0}\left(x_{0}, l\right) .
$$

Moreover, we have from Huisken's monotonicity formula [6] that

$$
\Theta_{t}\left(x_{0}, l\right) \leq \Theta_{0}\left(x_{0}, l+t\right) \text { for all } x_{0} \in \mathbb{C}^{n}, t>0, l>0 .
$$

We conclude this section with the following observation. Given $P_{1}, P_{2}$ transverse planes we have

$$
\Theta_{0}\left(x_{0}, l\right)=\int_{P_{1}+P_{2}} \Phi\left(x_{0}, l\right) \mathrm{d} \mathcal{H}^{n}<2 \text { for all } l>0
$$

unless $x_{0}=0$. One consequence of this observation is the following:

\section{Lemma 2.4}

- The self-expander $L$ is embedded.

- There is $c_{1}$ depending only on $L_{0}$ so that

$$
\mathcal{H}^{n}\left(L \cap B_{r}(x)\right) \leq c_{1} r^{n} \quad \text { for all } r>0 \text { and } x \in \mathbb{C}^{n} .
$$

Proof Suppose that $L$ is immersed. Then there exists $x_{0} \in L$ where

$$
\lim _{\delta \rightarrow 0} \Theta_{\frac{1}{2}}\left(x_{0}, \delta\right) \geq 2 .
$$

By (5), making $t=\frac{1}{2}$ and $l \rightarrow 0$, we obtain

$$
2 \leq \Theta_{0}\left(x_{0}, \frac{1}{2}\right)
$$

and so, by (6), $x_{0}=0$ and equality holds in the equation above. In this case,

$$
2 \leq \lim _{\delta \rightarrow 0} \Theta_{\frac{1}{2}}(0, \delta) \leq \Theta_{0}\left(x_{0}, \frac{1}{2}\right)=2
$$

and so equality holds in Huisken's monotonicity formula. Hence $L$ is also a selfshrinker (ie, $H=-x^{\perp}$ ) and thus $H=x^{\perp}=0$ as $L$ is a self-expander. Therefore, $L$ must be a cone but, because $L$ is asymptotic to $L_{0}$, this is only possible if $L=L_{0}$, which then contradicts the assumption that $L$ is smooth. This proves the first property. 
In what follows $c$ denotes a constant depending only on $n$. By (5) and (6)

$$
\frac{\mathcal{H}^{n}\left(L \cap B_{r}(x)\right)}{r^{n}} \leq c \Theta_{1 / 2}\left(x, r^{2}\right) \leq c \Theta_{0}\left(x, r^{2}+\frac{1}{2}\right) \leq 2 c .
$$

\section{Exponential decay}

In this section we show that the self-expander $L$ converges exponentially fast to $L_{0}$ outside a compact set. This naturally coincides with the behaviour of the relevant self-expanders in [10], but is in marked contrast to special Lagrangian Lawlor necks, which only converge at rate $O\left(r^{1-n}\right)$ to their asymptotic planes.

Let $G_{L}\left(n, \mathbb{C}^{n}\right)$ denote the set of all Lagrangian planes in $\mathbb{C}^{n}$, which has a natural topology when viewed as the homogeneous space $U(n) / O(n)$. Consider the open subset of $G_{L}\left(n, \mathbb{C}^{n}\right) \times G_{L}\left(n, \mathbb{C}^{n}\right)$ given by

$$
G_{n}=\left\{\left(P_{1}, P_{2}\right) \in G_{L}\left(n, \mathbb{C}^{n}\right) \times G_{L}\left(n, \mathbb{C}^{n}\right) \mid P_{1} \cap P_{2}=\{0\}\right\} .
$$

Given a compact set $K \subset G_{n}$, we denote by $\mathcal{S}(K)$ the set of all self-expanders which are asymptotic to $L_{0}=P_{1}+P_{2}$, with $\left(P_{1}, P_{2}\right) \in K$.

Theorem 3.1 For every compact set $K \subset G_{n}$ and $k \in \mathbb{N}$, there is $R_{1}>0, C$ and $b$ so that for all $L \in \mathcal{S}(K)$ we find $\psi \in C^{\infty}\left(L_{0} \backslash B_{R_{1}}\right)$ satisfying

$$
L \backslash B_{2 R_{1}} \subset\left\{x+J \bar{\nabla} \psi(x) \mid x \in L_{0} \backslash B_{R_{1}}\right\} \subset L \backslash B_{R_{1} / 2}
$$

and

$$
\|\psi\|_{C^{k}\left(L_{0} \backslash B_{R}\right)} \leq C e^{-b R^{2}} \text { for all } R \geq R_{1} .
$$

Proof The next proposition says that if $L$ is locally graphical over $L_{0} \backslash B_{R_{0}}$ for some $R_{0}$ and the local graph is asymptotic to zero in the $C^{k+1}$-norm, then we can find $R_{1}$ large so that $L$ is a global graph over $L_{0} \backslash B_{R_{1}}$ and the graph has its $C^{k}$-norm decaying exponentially fast.

Proposition 3.2 Fix $R_{0}>2 r_{0}>0, k \in \mathbb{N}$, a compact set $K \subset G_{n}$, and a decreasing function $D_{k}=D_{k}(r)$ tending to zero at infinity.

Suppose that for every $L \in \mathcal{S}(K)$ and $y_{0} \in L \backslash B_{R_{0}}$ we can find $x_{0} \in L_{0}$ and

$$
\phi: L_{0} \cap B_{2 r_{0}}\left(x_{0}\right) \rightarrow \mathbb{C}^{n}
$$

so that

- the $C^{k+1}\left(B_{2 r_{0}}\left(x_{0}\right)\right)$-norm of $\phi$ is bounded by $D_{k}\left(\left|y_{0}\right|\right)$,

- $L \cap \widehat{B}_{r_{0}}\left(y_{0}\right) \subset\left\{x+J \bar{\nabla} \phi(x) \mid x \in L_{0} \cap B_{2 r_{0}}\left(x_{0}\right)\right\}$, where $\widehat{B}_{r_{0}}\left(y_{0}\right)$ denotes the intrinsic ball of $L$ with radius $r_{0}$ and centered at $y_{0}$. 
Then there exist $R_{1}, C, b$, and an open set $B \subset \mathbb{C}^{n}$ with compact closure, depending on $r_{0}, R_{0}, D_{k}$ and $K$, such that for every $L \in \mathcal{S}(K)$ we can find $\psi \in C^{\infty}\left(L_{0} \backslash B_{R_{1}}\right)$ with

$$
L \backslash B \subset\left\{x+J \bar{\nabla} \psi(x) \mid x \in L_{0} \backslash B_{R_{1}}\right\}
$$

and

$$
\|\psi\|_{C^{k}\left(L_{0} \backslash B_{R}\right)} \leq C e^{-b R^{2}} \text { for all } R \geq R_{1} .
$$

Proof From the hypotheses of the proposition, for every $L \in \mathcal{S}(K)$ and $\widetilde{R}_{1}$ sufficiently large we can find an open set $\widetilde{B} \subset \mathbb{C}^{n}$ with compact closure and a projection map

$$
\pi_{L}: L \backslash \widetilde{B} \longrightarrow L_{0} \backslash B_{\widetilde{R}_{1}}
$$

We claim we can choose $R_{1}=R_{1}\left(r_{0}, R_{0}, C_{k}\right)$ so that $\pi_{L}$ is a diffeomorphism when restricted to $\pi_{L}^{-1}\left(L_{0} \backslash B_{R_{1}}\right)$.

Suppose not. Then we can find $L^{i} \in \mathcal{S}(K)$ with $L_{0}^{i}=P_{1}^{i}+P_{2}^{i}$ tending to $P_{1}+P_{2}$, $x_{i}$ in $L_{0}^{i} \backslash B_{R_{0}}$ such that $\left|x_{i}\right| \rightarrow \infty$ as $i \rightarrow \infty$, and $\pi_{L^{i}}^{-1}\left(x_{i}\right) \supset\left\{y_{i}, z_{i}\right\}$ where $y_{i} \neq z_{i}$ for all $i$.

By hypothesis, there exists $\delta_{0}>0$ such that $L^{i} \cap B_{2 \delta_{0}}\left(x_{i}\right)$ contains the graphs of functions $f_{i}, g_{i}$ on $B_{\delta_{0}}\left(x_{i}\right) \cap L_{0}^{i}$ with $f_{i}\left(x_{i}\right)=y_{i}$ and $g_{i}\left(x_{i}\right)=z_{i}$ for all $i$. Therefore, recalling $\Phi$ given in (2), Huisken's monotonicity formula implies

$$
\begin{aligned}
\int_{L_{0}^{i}} \Phi\left(x_{i}, \delta^{2}+\frac{1}{2}\right) \mathrm{d} \mathcal{H}^{n} & \geq \int_{L^{i}} \Phi\left(x_{i}, \delta^{2}\right) \mathrm{d} \mathcal{H}^{n} \\
& \geq \int_{L^{i} \cap B_{2 \delta_{0}}\left(x_{i}\right)} \Phi\left(x_{i}, \delta^{2}\right) \mathrm{d} \mathcal{H}^{n} \\
& \geq \int_{\operatorname{graph}\left(f_{i}\right) \cup \operatorname{graph}\left(g_{i}\right)} \Phi\left(x_{i}, \delta^{2}\right) \mathrm{d} \mathcal{H}^{n} \\
& \geq 2 \int_{B_{\delta_{0}}\left(x_{i}\right) \cap L_{0}^{i}} \Phi\left(x_{i}, \delta^{2}\right) \mathrm{d} \mathcal{H}^{n},
\end{aligned}
$$

where the last inequality comes from the fact that $L_{0}^{i}$ is a union of planes.

Since $L_{0}^{i}$ tends to $P_{1}+P_{2}$ with $P_{1} \cap P_{2}=\{0\}$ and $\left|x_{i}\right| \rightarrow \infty$, we have from the hypothesis of the proposition that $L_{0}^{i}-x_{i}$ tends to either $P_{1}$ or $P_{2}$. We assume that the first case occurs and so, after translating by $-x_{i}, B_{\delta_{0}}\left(x_{i}\right) \cap L_{0}^{i}$ tends to $B_{\delta_{0}}(0) \cap P_{1}$. Thus, letting $i \rightarrow \infty$ and then $\delta \rightarrow 0$, we obtain from (9) that

$$
1=\int_{P_{1}} \Phi\left(0, \frac{1}{2}\right) \mathrm{d} \mathcal{H}^{n} \geq 2
$$


This proves the claim.

The fact that $\pi_{L}$ is a diffeomorphism implies the existence of a smooth vector field $X$ on $L_{0} \backslash B_{R_{1}}$ that is tangent to $L_{0} \backslash B_{R_{1}}$ so that, for some open set $B$,

$$
L \backslash B=\left\{x+J X(x): x \in L_{0} \backslash B_{R_{1}}\right\} .
$$

Since $L$ is Lagrangian, it is standard to see that $X=\bar{\nabla} \psi$ locally. We need to make sure that $\psi$ is defined globally on $L_{0} \backslash B_{R_{1}}$. Recall the primitive $\beta$ for the Liouville form $\lambda$ on $L$. Set $\bar{\beta}=\beta \circ \pi_{L}^{-1}$ and define $\psi$ on $L_{0} \backslash B_{R_{1}}$ by

$$
\psi(x)=\frac{1}{2}(\langle X(x), x\rangle-\bar{\beta}(x)),
$$

using the standard Euclidean inner product $\langle\cdot, \cdot\rangle$. Then, for every vector $v$ tangent to $L_{0}$ we have

$$
\begin{aligned}
\langle\bar{\nabla} \psi(x), v\rangle & =\frac{1}{2}\left(\left\langle\bar{\nabla}_{v} X, x\right\rangle+\langle X(x), v\rangle-\langle\bar{\nabla} \bar{\beta}(x), v\rangle\right) \\
& =\frac{1}{2}\left(\left\langle\bar{\nabla}_{v} X, x\right\rangle+\langle X(x), v\rangle-\lambda\left(v+J \bar{\nabla}_{v} X(x)\right)\right)
\end{aligned}
$$

since $\bar{\nabla} \bar{\beta}(x)=\bar{\nabla} \beta(x+J X(x))$ and $\mathrm{d} \beta=\left.\lambda\right|_{L}$, and hence

$$
\begin{aligned}
\langle\bar{\nabla} \psi(x), v\rangle & =\frac{1}{2}\left(\left\langle\bar{\nabla}_{v} X, x\right\rangle+\langle X(x), v\rangle-\left\langle J x-X(x), v+J \bar{\nabla}_{v} X(x)\right\rangle\right) \\
& =\langle X(x), v\rangle-\frac{1}{2}\langle J x, v\rangle+\frac{1}{2}\left\langle X(x), J \bar{\nabla}_{v} X(x)\right\rangle .
\end{aligned}
$$

Observe that $\langle J x, v\rangle=0$ since $L_{0}$ is a pair of Lagrangian planes and thus $x$ is tangent to $L_{0}$ and orthogonal to $J v$. Furthermore, $\left\langle X(x), J \bar{\nabla}_{v} X(x)\right\rangle=0$ because $\bar{\nabla}_{v} X$ has no component orthogonal to $L_{0}$ as $L_{0}$ is a union of planes. Thus $\bar{\nabla} \psi=X$, the decomposition (7) holds, and for $R \geq R_{1}$,

$$
\|\nabla \psi\|_{C^{k+1}\left(L_{0} \backslash B_{R}\right)} \leq D_{k}(R-1),
$$

where $D_{k}$ is our given decreasing function by hypothesis.

We now wish to prove (8). Let $S_{0}$ be a connected component of $L_{0} \backslash\{0\}$, ie, $S_{0}$ is a Lagrangian plane minus the origin, and let $S$ be the connected component of $L \backslash B_{R_{1}}$ asymptotic to $S_{0}$ given by the graphical decomposition in (7). After changing coordinates so that $S_{0}$ is identified with a real plane, we consider the following vectorvalued function defined on $S$ :

$$
\boldsymbol{y}=(J \bar{\nabla} \psi) \circ \pi_{L}=i \sum_{j=1}^{n} y_{j} \frac{\partial}{\partial y_{j}} \in \mathbb{C}^{n} .
$$


Let $\Delta=-\mathrm{d}^{*} \mathrm{~d}$ be the analyst's Laplacian (or Laplace-Beltrami operator) acting on functions on $L$. For $j=1, \ldots, n$ we have on $S$ that

$$
\Delta y_{j}^{2}=2 y_{j}\left\langle i \frac{\partial}{\partial y_{j}}, H\right\rangle+2\left|\partial_{y_{j}}^{\top}\right|^{2} \Longrightarrow \Delta|\boldsymbol{y}|^{2} \geq 2\langle\boldsymbol{y}, H\rangle \text {. }
$$

Calculating with respect to the induced metric on $S$ and recalling that $\boldsymbol{x}$ is the position vector on $L$, we see that, since $L$ is a self-expander,

$$
|\boldsymbol{y}|^{2}=\langle\boldsymbol{y}, \boldsymbol{x}\rangle=\left\langle\boldsymbol{y}, \boldsymbol{x}^{\perp}\right\rangle+\left\langle\boldsymbol{y}, \boldsymbol{x}^{\top}\right\rangle=\langle\boldsymbol{y}, H\rangle+\left\langle\boldsymbol{y}, \boldsymbol{x}^{\top}\right\rangle=\langle\boldsymbol{y}, H\rangle+\frac{1}{2}\left\langle\nabla|\boldsymbol{y}|^{2}, \boldsymbol{x}\right\rangle .
$$

Thus, if we define

$$
\mathcal{L}(\phi)=\Delta \phi+\langle\boldsymbol{x}, \nabla \phi\rangle-2 \phi
$$

for suitably differentiable functions $\phi$ on $S$, where all quantities are computed with respect to the induced metric on $S$, we see that

$$
\mathcal{L}\left(|\boldsymbol{y}|^{2}\right) \geq 0
$$

We are now in the position to construct a barrier for $|\boldsymbol{y}|^{2}$ and deduce (8).

Set $\rho(x)=\exp \left(-|x|^{2} / 2\right)$, an ambient function on $\mathbb{C}^{n}$. Since $L$ is a self-expander, we have that

$$
\Delta\left(|x|^{2}\right)=2 n+2\langle H, x\rangle=2 n+2\left|x^{\perp}\right|^{2} .
$$

Therefore one sees that, on $L$,

$$
\Delta \rho=-\frac{\rho}{2} \Delta\left(|x|^{2}\right)+\left.\left.\frac{\rho}{4}|\nabla| x\right|^{2}\right|^{2}=\rho\left(\left|x^{\top}\right|^{2}-n-\left|x^{\perp}\right|^{2}\right) .
$$

Moreover,

$$
\langle\nabla \rho, \boldsymbol{x}\rangle=-\rho\left|\boldsymbol{x}^{\top}\right|^{2}
$$

and so

$$
\mathcal{L}(\rho)=\rho\left(\left|\boldsymbol{x}^{\top}\right|^{2}-n-2-|\boldsymbol{x}|^{2}\right) \leq-(n+2) \rho .
$$

Let $\varepsilon>0$ and using (10) choose $C=C\left(r_{0}, R_{0}, D_{k}\right)$ so that

$$
|\boldsymbol{y}|^{2}=\left|(\bar{\nabla} \psi) \circ \pi_{L}\right|^{2}<C \exp \left(-|x|^{2} / 2\right) \quad \text { on } \partial S .
$$

Set $\tilde{\rho}=\varepsilon+C \rho$. For all $R$ sufficiently large we have $|\boldsymbol{y}|^{2}<\tilde{\rho}$ on $S \cap \partial\left\{R_{1}<|x|<R\right\}$ because $|\boldsymbol{y}|^{2}$ tends to zero at infinity by hypothesis. Furthermore, using $\mathcal{L}\left(|\boldsymbol{y}|^{2}\right) \geq 0$ and (12), we have

$$
\mathcal{L}\left(\widetilde{\rho}-|\boldsymbol{y}|^{2}\right)=\mathcal{L}\left(\rho-|\boldsymbol{y}|^{2}\right)-2 \varepsilon<0
$$


and thus the maximum principle implies that

$$
|\boldsymbol{y}|^{2}<\varepsilon+C \exp \left(-|x|^{2} / 2\right) \text { on } S \cap\left\{R_{1}<|x|<R\right\} .
$$

Letting $R \rightarrow \infty$ and then $\varepsilon \rightarrow 0$, we conclude that

$$
|\boldsymbol{y}|^{2} \leq C \exp \left(-|x|^{2} / 2\right) \quad \text { on } S .
$$

Recall that on $S$ we have $\boldsymbol{y}=(J \bar{\nabla} \psi) \circ \pi_{L}$. Therefore we can add a constant to $\psi$ and find some other constant $C=C\left(r_{0}, R_{0}, D_{k}\right)$ so that, after integration,

$$
|\psi(x)| \leq C \exp \left(-\frac{1}{4}|x|^{2}\right) \text { for all } x \in S_{0} \backslash B_{R_{1}} .
$$

As the $C^{k+1}$ norm of $\psi$ in $S_{0} \backslash B_{R_{1}}$ is bounded, it follows from standard interpolation inequalities for Hölder spaces (see eg Krylov [11, Theorem 3.2.1]) that, for some further constant $C=C\left(r_{0}, R_{0}, D_{k}\right)$,

$$
\|\psi\|_{C^{k}\left(L_{0} \backslash B_{R}\right)} \leq C \exp \left(-a R^{2}\right)
$$

for some constant $a>0$ and any $R \geq R_{1}$.

We can argue in the same manner for each connected component of $L_{0} \backslash B_{R_{1}}$ and conclude the desired result.

We now make an observation concerning $\Theta$ given in (3). Given $L^{i} \in \mathcal{S}(K)$, we denote by $\Theta_{t}^{i}\left(x_{0}, l\right)$ the Gaussian density ratios (3) evaluated at $L_{t}^{i}$.

Lemma 3.3 Let $L^{i}$ be a sequence in $\mathcal{S}(K)$ and $\left(x_{i}\right)_{i \in \mathbb{N}}$ a sequence of points in $\mathbb{C}^{n}$ with $\left|x_{i}\right|$ tending to infinity. Then, for all $l>0$,

$$
\lim _{i \rightarrow \infty} \Theta_{0}^{i}\left(x_{i}, l\right) \leq 1
$$

with equality only if $\lim _{i \rightarrow \infty} \operatorname{dist}\left(x_{i}, L_{0}^{i}\right)=0$.

Proof We have $L_{0}^{i}=P_{1}^{i}+P_{2}^{i}$ and write $x_{i}=a_{i}^{1}+b_{i}^{1}=a_{i}^{2}+b_{i}^{2}$, where $a_{i}^{j} \in P_{j}^{i}$ and $\left\langle a_{i}^{j}, b_{i}^{j}\right\rangle=0$ for $j=1,2$. We set $Q_{i}=L_{0}^{i}-x_{i}$, where we mean that we translate $L_{0}^{i}$ by the vector given by $x_{i}$. We have

$$
\min \left\{\left|b_{i}^{1}\right|,\left|b_{i}^{2}\right|\right\}=\operatorname{dist}\left(x_{i}, L_{0}^{i}\right)=\operatorname{dist}\left(0, Q_{i}\right) .
$$

Suppose first that $\limsup _{i \rightarrow \infty}\left|b_{i}^{1}\right|=\limsup _{i \rightarrow \infty}\left|b_{i}^{2}\right|=+\infty$. We then have $\operatorname{dist}\left(0, Q_{i}\right)$ tending to infinity and so

$$
\lim _{i \rightarrow \infty} \Theta_{0}\left(x_{i}, l\right)=\lim _{i \rightarrow \infty} \int_{Q_{i}} \Phi(0, l) \mathrm{d} \mathcal{H}^{n}=0 .
$$


Otherwise, without loss of generality, $\lim _{\sup } \operatorname{su}_{i \rightarrow \infty}\left|b_{i}^{1}\right|<\infty$, and necessarily $\liminf _{i \rightarrow \infty}\left|a_{i}^{1}\right|=+\infty$. Note that we must also have $\liminf _{i \rightarrow \infty}\left|b_{i}^{2}\right|=+\infty$ because otherwise we could extract a subsequence of $\left(P_{1}^{i}, P_{2}^{i}\right)$ converging to a pair of planes intersecting along a line.

Therefore $Q_{i}$ sequentially converges to $P_{1}+b$, ie, an affine plane parallel to some plane $P_{1}$, where $b=\lim _{i \rightarrow \infty} b_{i}^{1}$ is orthogonal to $P_{1}$. Thus

$$
\begin{aligned}
\lim _{i \rightarrow \infty} \Theta_{0}^{i}\left(x_{i}, l\right) & =\int_{P_{1}+b} \Phi(0, l) \mathrm{d} \mathcal{H}^{n}=\int_{P_{1}} \Phi(-b, l) \mathrm{d} \mathcal{H}^{n} \\
& =\exp \left(-\frac{|b|^{2}}{4 l}\right) \int_{P_{1}} \Phi(0, l) \mathrm{d} \mathcal{H}^{n}=\exp \left(-\frac{|b|^{2}}{4 l}\right) \leq 1,
\end{aligned}
$$

with equality only if $b=0$. This proves the desired result.

We can now finish the proof of Theorem 3.1. The idea is to show that the hypotheses of Proposition 3.2 are satisfied for all $L \in \mathcal{S}(K)$.

First we claim that

$$
\lim _{R \rightarrow \infty} \sup \left\{\operatorname{dist}\left(x, L_{0}\right) \mid L \in \mathcal{S}(K), x \in L \backslash B_{R}\right\}=0 .
$$

Indeed, if we choose any sequence $L^{i} \in \mathcal{S}(K)$ and pick $x_{i} \in L^{i}$ with $\left|x_{i}\right|$ tending to infinity, we have from (5) that

$$
1=\lim _{r \rightarrow 0} \Theta_{1 / 2}^{i}\left(x_{i}, r\right) \leq \lim _{r \rightarrow 0} \Theta_{0}^{i}\left(x_{i}, r+\frac{1}{2}\right)=\Theta_{0}^{i}\left(x_{i}, \frac{1}{2}\right)
$$

and thus

$$
\lim _{i \rightarrow \infty} \Theta_{0}^{i}\left(x_{i}, \frac{1}{2}\right) \geq 1 \text {. }
$$

The claim now follows from Lemma 3.3.

Second we claim the existence of $R_{1}>0$ so that the $C^{2, \alpha}$ norm of $L \backslash B_{R_{1}}$ is uniformly bounded for all $L \in \mathcal{S}(K)$. Indeed, with $\varepsilon_{0}>0$ fixed, we obtain from Lemma 3.3 the existence of $R_{1}$ so that for all $L \in \mathcal{S}(K)$ we have

$$
\Theta_{0}(x, 2) \leq 1+\varepsilon_{0} \quad \text { for all }|x| \geq R_{1} / 2
$$

and so, for all $t, l \in[0,1]$ we have

$$
\Theta_{t}(x, l) \leq \Theta_{0}(x, l+t) \leq \Theta_{0}(x, 2) \leq 1+\varepsilon_{0} \text { for all }|x| \geq R_{1} / 2 .
$$

White's Regularity Theorem [23, Theorem 3.1] implies the desired claim.

From the first and second claim we see that given $r>0$ and $\varepsilon>0$ we can find $R_{2}$ so that for all $L \in \mathcal{S}(K)$ and $x \in L \backslash B_{R_{2}}$ we have that $\widehat{B}_{r}(x) \cap L$ is $\varepsilon$-close in $C^{2, \alpha}$ to 
a ball of radius $r$ in $L_{0}$. Elliptic regularity implies that for every $k \in \mathbb{N}$ we can choose $R_{2}$ larger so that $\widehat{B}_{r}(x) \cap L$ is $\varepsilon$-close in $C^{k+1, \alpha}$ to a ball of radius $r$ in $L_{0}$. Thus, for every $k \in \mathbb{N}$, we can find $r_{0}, R_{0}$, and $D_{k}$ so that the hypotheses of Proposition 3.2 are satisfied for all $L \in \mathcal{S}(K)$. This implies the desired result.

\section{Fredholm theory}

In this section we develop the Fredholm theory for the operator

$$
\mathcal{L}(\phi)=\Delta \phi+\langle\boldsymbol{x}, \nabla \phi\rangle-2 \phi
$$

defined on the self-expander $L$, which already arose in (11). The relevant spaces to consider are given below.

Definition 4.1 For $k \in \mathbb{Z}^{+}$, let $H^{k}(L)$ denote the Sobolev space $W^{k, 2}(L)$ with norm

$$
\|\phi\|_{H^{k}}=\left(\sum_{j=0}^{k} \int_{L}\left|\nabla^{j} \phi\right|^{2} \mathrm{~d} \mathcal{H}^{n}\right)^{\frac{1}{2}} .
$$

Let $H_{*}^{k}(L)$ denote the subspace of $H^{k}(L)$ such that the norm

$$
\|\phi\|_{H_{*}^{k}}=\left(\sum_{j=0}^{k} \int_{L}\left|\nabla^{j} \phi\right|^{2} \mathrm{~d} \mathcal{H}^{n}+\sum_{j=1}^{k-1} \int_{L}\left\langle\boldsymbol{x}^{\top}, \nabla^{j} \phi\right\rangle^{2} \mathrm{~d} \mathcal{H}^{n}\right)^{\frac{1}{2}}
$$

is finite. Here the symbol $\left\langle\boldsymbol{x}^{\top}, \nabla^{j} \phi\right\rangle$ means that we form a tensor by contracting $\boldsymbol{x}^{\top}$ and $\nabla^{j} \phi$ using the Euclidean metric; for example, $\left\langle\boldsymbol{x}^{\top}, \nabla \phi\right\rangle$ is given by viewing $\nabla \phi$ as a vector using the Euclidean metric and then taking its inner product with $\boldsymbol{x}^{\top}$. Thus $\left\langle\boldsymbol{x}^{\top}, \nabla^{j} \phi\right\rangle^{2}$ is the square norm of the tensor $\left\langle\boldsymbol{x}^{\top}, \nabla^{j} \phi\right\rangle$. Both $H^{k}$ and $H_{*}^{k}$ are Banach spaces (in fact, Hilbert spaces).

Our first result, which shows the utility of Definition 4.1, is the following.

Proposition 4.2 The map $\mathcal{L}: H_{*}^{k+2}(L) \rightarrow H^{k}(L)$ is well-defined and continuous.

Proof From Theorem 3.1 we have that every derivative of the second fundamental form of $L$ is uniformly bounded. Thus if $T$ is a tensor on $L$ we have from the Bochner formula and Gauss equation that

$$
\nabla \Delta T=\Delta \nabla T+C_{1} \star \nabla T+C_{0} \star T
$$


where $C_{0}, C_{1}$ are two uniformly bounded tensors (depending only on $L$ ) and $A \star B$ denotes any contraction of tensor $A$ with tensor $B$. Moreover, using the fact that $H=\boldsymbol{x}^{\perp}$ on $L$ we also have

$$
\nabla\left\langle\boldsymbol{x}^{\top}, T\right\rangle=\left\langle\boldsymbol{x}^{\top}, \nabla T\right\rangle+C_{0} \star T,
$$

where $C_{0}$ is another uniformly bounded tensor which depends only on $L$.

We can use (15) and (16) inductively to conclude that for all $i \in \mathbb{N}$ and $\phi \in C_{0}^{\infty}(L)$ we have

$$
\nabla^{i} \mathcal{L}(\phi)=\Delta \nabla^{i} \phi+\left\langle\boldsymbol{x}^{\top}, \nabla^{i+1} \phi\right\rangle+\sum_{j=0}^{i} C_{j} \star \nabla^{j} \phi,
$$

where the $C_{j}$ are uniformly bounded tensors. Thus for all $i \in \mathbb{N}$ we can find a positive constant $c_{i}=c_{i}(L)$ so that

$$
\int_{L}\left|\nabla^{i} \mathcal{L}(\phi)\right|^{2} \mathrm{~d} \mathcal{H}^{n} \leq c_{i}\left(\sum_{j=0}^{i+2} \int_{L}\left|\nabla^{j} \phi\right|^{2} \mathrm{~d} \mathcal{H}^{n}+\int_{L}\left\langle\boldsymbol{x}^{\top}, \nabla^{i+1} \phi\right\rangle^{2} \mathrm{~d} \mathcal{H}^{n}\right) .
$$

It follows that $\mathcal{L}$ defines a continuous map from $H_{*}^{k+2}(L)$ to $H^{k}(L)$.

Theorem 4.3 The map $\mathcal{L}: H_{*}^{2}(L) \longrightarrow L^{2}(L)$ is an isomorphism.

Proof We start by proving the existence of a positive constant $C_{0}=C_{0}(L, n)$ so that for all $\phi$ in $H_{*}^{2}(L)$ we have

$$
\|\phi\|_{H_{*}^{2}}^{2} \leq C_{0} \int_{L}|\mathcal{L}(\phi)|^{2} \mathrm{~d} \mathcal{H}^{n}=C_{0}\|\mathcal{L}(\phi)\|_{L^{2}}^{2} .
$$

Using the fact that $H=x^{\perp}$ on $L$, we have

$$
\operatorname{div} \boldsymbol{x}^{\top}=n+\left|\boldsymbol{x}^{\perp}\right|^{2}
$$

and so direct computation shows that

$$
2 \phi\langle\boldsymbol{x}, \nabla \phi\rangle=\operatorname{div}\left(\boldsymbol{x}^{\top} \phi^{2}\right)-\left(n+\left|\boldsymbol{x}^{\perp}\right|^{2}\right) \phi^{2} .
$$

Thus

$$
-\int_{L} \mathcal{L}(\phi) \phi \mathrm{d} \mathcal{H}^{n}=\int_{L}|\nabla \phi|^{2}+\left(2+n / 2+\left|x^{\perp}\right|^{2} / 2\right) \phi^{2} \mathrm{~d} \mathcal{H}^{n}
$$

and so, since $-\mathcal{L}(\phi) \phi \leq|\mathcal{L}(\phi)|^{2}+\frac{1}{4} \phi^{2}$, we obtain

$$
\int_{L}|\nabla \phi|^{2} \mathrm{~d} \mathcal{H}^{n} \leq \int_{L}|\mathcal{L}(\phi)|^{2} \mathrm{~d} \mathcal{H}^{n}
$$


Moreover, if $A$ is the second fundamental form of $L$,

$$
\begin{aligned}
\Delta \phi\langle\boldsymbol{x}, \nabla \phi\rangle=\operatorname{div}\left(\nabla \phi\left\langle\boldsymbol{x}^{\top}, \nabla \phi\right\rangle\right. & \left.-\boldsymbol{x}^{\top} \frac{|\nabla \phi|^{2}}{2}\right) \\
& +\frac{|\nabla \phi|^{2}}{2}\left(n-2+\left|\boldsymbol{x}^{\perp}\right|^{2}\right)-\langle H, A(\nabla \phi, \nabla \phi)\rangle
\end{aligned}
$$

and combining (22) with (20) we obtain

$$
\begin{aligned}
\int_{L}|\mathcal{L}(\phi)|^{2} \mathrm{~d} \mathcal{H}^{n} & =\int_{L}(\Delta \phi)^{2}+\langle\boldsymbol{x}, \nabla \phi\rangle^{2}+\left(n+2+\left|\boldsymbol{x}^{\perp}\right|^{2}\right)|\nabla \phi|^{2} \mathrm{~d} \mathcal{H}^{n} \\
& +2 \int_{L}\left(n+2+\left|\boldsymbol{x}^{\perp}\right|^{2}\right) \phi^{2} \mathrm{~d} \mathcal{H}^{n}-2 \int_{L}\langle H, A(\nabla \phi, \nabla \phi)\rangle \mathrm{d} \mathcal{H}^{n} .
\end{aligned}
$$

From the Bochner formula and Gauss equation there is a constant $c=c(n)$ so that on $L$ we have

$$
|\nabla \Delta \phi-\Delta \nabla \phi| \leq c|A|^{2}|\nabla \phi|
$$

Observing that

$$
\begin{gathered}
\operatorname{div}\left(\nabla^{2} \phi(\nabla \phi, \cdot)\right)=\langle\Delta \nabla \phi, \nabla \phi\rangle+\left|\nabla^{2} \phi\right|^{2}, \\
\operatorname{div}(\Delta \phi \nabla \phi)=\langle\nabla \Delta \phi, \nabla \phi\rangle+(\Delta \phi)^{2},
\end{gathered}
$$

we thus see that

$$
\left|\nabla^{2} \phi\right|^{2} \leq(\Delta \phi)^{2}+\operatorname{div}\left(\nabla^{2} \phi(\nabla \phi, \cdot)-\Delta \phi \nabla \phi\right)+c|A|^{2}|\nabla \phi|^{2} .
$$

Inserting (24) in (23), we have

$$
\begin{aligned}
\int_{L}\left|\nabla^{2} \phi\right|^{2}+\langle\boldsymbol{x}, \nabla \phi\rangle^{2}+|\nabla \phi|^{2} & +\phi^{2} \mathrm{~d} \mathcal{H}^{n} \\
& \leq \int_{L}|\mathcal{L}(\phi)|^{2} \mathrm{~d} \mathcal{H}^{n}+(c+2) \int_{L}|A|^{2}|\nabla \phi|^{2} \mathrm{~d} \mathcal{H}^{n} .
\end{aligned}
$$

From Theorem 3.1 we know that $|A|^{2}$ is uniformly bounded on $L$ and so we have from (21) the existence of a constant $C=C(L, n)$ so that

$$
\int_{L}|A|^{2}|\nabla \phi|^{2} \mathrm{~d} \mathcal{H}^{n} \leq C \int_{L}|\mathcal{L}(\phi)|^{2} \mathrm{~d} \mathcal{H}^{n} .
$$

This last inequality and (25) imply (18) at once.

The immediate consequence of (18) is that $\mathcal{L}$ is injective. It also follows that its range is closed for the following reason. If $v_{i}=\mathcal{L}\left(\phi_{i}\right)$ is a sequence converging in $L^{2}(L)$ to $v$, then $(18)$ implies $\left(\phi_{i}\right)_{i \in \mathbb{N}}$ is a Cauchy sequence in $H_{*}^{2}(L)$ and thus 
sequentially converging to $\phi \in H_{*}^{2}(L)$. Naturally, $\mathcal{L}(\phi)=v$ since $\mathcal{L}$ is continuous by Proposition 4.2.

We now argue that $\mathcal{L}$ is surjective. In order to do so we compute the formal adjoint $\mathcal{L}^{*}$ of $\mathcal{L}$. Using (19) we have, for every $u, v \in C^{\infty}(L)$,

$$
\langle\boldsymbol{x}, \nabla u\rangle v=\operatorname{div}\left(\boldsymbol{x}^{\top} u v\right)-\left(n+\left|\boldsymbol{x}^{\perp}\right|^{2}\right) u v-\langle\boldsymbol{x}, \nabla v\rangle u .
$$

Hence, if $\phi \in C_{0}^{\infty}(L)$ and $\eta \in C^{\infty}(L)$,

$$
\int_{L} \mathcal{L}(\phi) \eta \mathrm{d} \mathcal{H}^{n}=\int_{L} \phi\left(\Delta \eta-\langle\boldsymbol{x}, \nabla \eta\rangle-\left(n+\left|\boldsymbol{x}^{\perp}\right|^{2}+2\right) \eta\right) \mathrm{d} \mathcal{H}^{n}
$$

which means

$$
\mathcal{L}^{*}(\eta)=\Delta \eta-\langle\boldsymbol{x}, \nabla \eta\rangle-\left(n+\left|\boldsymbol{x}^{\perp}\right|^{2}+2\right) \eta
$$

Suppose that $\mathcal{L}$ is not surjective. Since its range is closed we can find $\eta \in L^{2}(L)$ non-zero such that

$$
\int_{L} \mathcal{L}(\phi) \eta \mathrm{d} \mathcal{H}^{n}=0 \quad \text { for all } \phi \in C_{0}^{\infty}(L)
$$

Elliptic regularity implies that $\eta$ is smooth and hence a solution to $\mathcal{L}^{*}(\eta)=0$. The next lemma implies that $\eta=0$ which is a contradiction.

Lemma 4.4 If $\eta \in C^{\infty}(L) \cap L^{2}(L)$ and $\mathcal{L}^{*}(\eta)=0$, then $\eta=0$.

Proof We start by arguing that $\eta \in H^{1}(L)$. For each $R>0$ consider a cut-off function $\phi_{R} \in C_{0}^{\infty}\left(\mathbb{C}^{n}\right)$ so that

$$
0 \leq \phi_{R} \leq 1,\left.\quad \phi_{R}\right|_{B_{R}}=1, \quad \operatorname{supp}\left(\phi_{R}\right) \subseteq B_{2 R}, \quad\left|\nabla \phi_{R}\right| \leq c_{0} R^{-1},
$$

for some universal constant $c_{0}$.

Using (26) with $u=\eta^{2} / 2$ and $v=\phi_{R}^{2}$ we have

$$
\begin{aligned}
0=-\int_{L} \mathcal{L}^{*}(\eta) \eta \phi_{R}^{2} \mathrm{~d} \mathcal{H}^{n}=\int_{L}|\nabla \eta|^{2} & \phi_{R}^{2}+\left(\frac{n+\left|\boldsymbol{x}^{\perp}\right|^{2}}{2}+2\right) \eta^{2} \phi_{R}^{2} \mathrm{~d} \mathcal{H}^{n} \\
& +\int_{L}\left\langle\nabla \eta, \nabla \phi_{R}^{2}\right\rangle \eta \mathrm{d} \mathcal{H}^{n}-\int_{L}\left\langle\boldsymbol{x}, \nabla \phi_{R}^{2}\right\rangle \frac{\eta^{2}}{2} \mathrm{~d} \mathcal{H}^{n} .
\end{aligned}
$$

We have for some universal constant $c$ and all $\varepsilon>0$ that

$$
\int_{L}\left\langle\nabla \eta, \nabla \phi_{R}^{2}\right\rangle \eta \mathrm{d} \mathcal{H}^{n} \leq c \varepsilon \int_{L}|\nabla \eta|^{2} \phi_{R}^{2} \mathrm{~d} \mathcal{H}^{n}+\frac{c}{\varepsilon} \int_{L} \eta^{2} \mathrm{~d} \mathcal{H}^{n}
$$


and thus we find another uniform constant $c$ so that

$$
\int_{L}|\nabla \eta|^{2} \phi_{R}^{2} \mathrm{~d} \mathcal{H}^{n} \leq c \int_{L}\left\langle\boldsymbol{x}, \nabla \phi_{R}^{2}\right\rangle \frac{\eta^{2}}{2} \mathrm{~d} \mathcal{H}^{n}+c \int_{L} \eta^{2} \mathrm{~d} \mathcal{H}^{n}
$$

The term $\left\langle\boldsymbol{x}, \nabla \phi_{R}^{2}\right\rangle$ is uniformly bounded (independent of $R$ ) by (28) and so

$$
\int_{L}|\nabla \eta|^{2} \phi_{R}^{2} \mathrm{~d} \mathcal{H}^{n} \leq c \int_{L} \eta^{2} \mathrm{~d} \mathcal{H}^{n}
$$

for some other universal constant $c$. Letting $R$ tend to infinity we obtain that $\eta \in$ $H^{1}(L)$.

We now show that $\eta=0$. Set

$$
f(r)=\int_{L \cap B_{r}} \eta^{2} \mathrm{~d} \mathcal{H}^{n}+\left(2+\frac{n}{2}\right)^{-1} \int_{L \cap B_{r}}|\nabla \eta|^{2} \mathrm{~d} \mathcal{H}^{n} .
$$

The idea is to show that

$$
\left(2+\frac{n}{2}\right) f(r) \leq r f^{\prime}(r) \text { for almost all } r \geq 2+\frac{n}{2} .
$$

If true, we obtain from integrating (29) that, for all $r \geq r_{1} \geq 2+\frac{n}{2}$,

$$
f(r) \geq f\left(r_{1}\right)\left(\frac{r}{r_{1}}\right)^{2+(n / 2)} .
$$

As $\eta \in H^{1}(L)$ the function $f$ is uniformly bounded, which contradicts the inequality above unless $f \equiv 0$, which means $\eta=0$.

Hence to complete the proof we need to show (29). Applying integration by parts to the identity

$$
\int_{L \cap B_{r}} \mathcal{L}^{*}(\eta) \eta \mathrm{d} \mathcal{H}^{n}=0
$$

and using (20) with $\phi=\eta$ we obtain

$$
\int_{L \cap B_{r}}|\nabla \eta|^{2}+\left(2+\frac{n}{2}+\frac{\left|x^{\perp}\right|^{2}}{2}\right) \eta^{2} \mathrm{~d} \mathcal{H}^{n}=-\frac{1}{2} \oint_{\partial\left(L \cap B_{r}\right)}\left(\left\langle\boldsymbol{x}^{\top}, \nu\right\rangle \eta^{2}-\partial_{\nu} \eta^{2}\right) \mathrm{d} \mathcal{H}^{n-1},
$$

where $v$ is the outward unit normal to the boundary of $L \cap B_{r}$. Therefore

$$
\begin{aligned}
\left(2+\frac{n}{2}\right) f(r) & \leq \frac{r}{2} \oint_{\partial\left(L \cap B_{r}\right)} \eta^{2} \mathrm{~d} \mathcal{H}^{n-1}+\oint_{\partial\left(L \cap B_{r}\right)}|\eta||\nabla \eta| \mathrm{d} \mathcal{H}^{n-1} \\
& \leq \frac{r+1}{2} \oint_{\partial\left(L \cap B_{r}\right)} \eta^{2} \mathrm{~d} \mathcal{H}^{n-1}+\frac{1}{2} \oint_{\partial\left(L \cap B_{r}\right)}|\nabla \eta|^{2} \mathrm{~d} \mathcal{H}^{n-1}
\end{aligned}
$$


On the other hand using the co-area formula we have, for almost all $r$,

$$
\begin{aligned}
f^{\prime}(r) & =\oint_{\partial\left(L \cap B_{r}\right)} \eta^{2} \frac{|\boldsymbol{x}|}{\left|\boldsymbol{x}^{\top}\right|} \mathrm{d} \mathcal{H}^{n-1}+\left(2+\frac{n}{2}\right)^{-1} \oint_{\partial\left(L \cap B_{r}\right)}|\nabla \eta|^{2} \frac{|\boldsymbol{x}|}{\left|\boldsymbol{x}^{\top}\right|} \mathrm{d} \mathcal{H}^{n-1} \\
& \geq \oint_{\partial\left(L \cap B_{r}\right)} \eta^{2} \mathrm{~d} \mathcal{H}^{n-1}+\left(2+\frac{n}{2}\right)^{-1} \oint_{\partial\left(L \cap B_{r}\right)}|\nabla \eta|^{2} \mathrm{~d} \mathcal{H}^{n-1} .
\end{aligned}
$$

Combining this inequality with (30) we obtain that (29) holds for almost all $r \geq 2+\frac{n}{2}$.

Applying Lemma 4.4 shows that $\mathcal{L}$ is surjective, completing the proof of Theorem 4.3.

Corollary 4.5 The map $\mathcal{L}: H_{*}^{k+2}(L) \longrightarrow H^{k}(L)$ is an isomorphism for all $k \in \mathbb{N}$.

Proof We proceed by induction where the case $k=0$ follows from Theorem 4.3. Assume Corollary 4.5 holds for some $k \in \mathbb{N}$. Thus $\mathcal{L}$ is injective and given $v \in$ $H^{k+1}(L) \subset H^{k}(L)$ there is $\phi \in H_{*}^{k+2}(L)$ so that $\mathcal{L}(\phi)=v$. We need to show that $\phi \in H_{*}^{k+3}(L)$ in order to prove the corollary.

Set $T=\nabla^{k+1} \phi$ and recall that $\Delta$ acting on $T$ is given by the trace of $\nabla^{2} T$. From (17) there exists a constant $C_{1}=C_{1}(L, n, k)$ so that

$$
\left\|\Delta T+\left\langle\boldsymbol{x}^{\top}, \nabla T\right\rangle\right\|_{L^{2}}^{2} \leq C_{1}\left(\|\phi\|_{H_{*}^{k+1}}^{2}+\|\mathcal{L}(\phi)\|_{H^{k+1}}^{2}\right) .
$$

Next we argue that, for some constant $C_{2}=C_{2}(L, n, k)$,

$$
\left|\int_{L}\left\langle\Delta T, \nabla_{\boldsymbol{x} \top} T\right\rangle \mathrm{d} \mathcal{H}^{n}\right| \leq C_{2} \int_{L}|\nabla T|^{2}+|T|^{2} \mathrm{~d} \mathcal{H}^{n} .
$$

Reasoning as in (22), there is a universal constant $c=c(n, k)$ so that

$$
\begin{aligned}
\mid\left\langle\Delta T, \nabla_{\left.\boldsymbol{x}^{\top} T\right\rangle-\operatorname{div}(}\left(\left\langle\nabla T, \nabla_{\boldsymbol{x}^{\top}}\right.\right.\right. & \left.T\rangle-\boldsymbol{x}^{\top} \frac{|\nabla T|^{2}}{2}\right) \mid \\
& \leq c|\nabla T|^{2}\left(1+|A|^{2}+\left|\boldsymbol{x}^{\perp}\right|^{2}\right)+c|T||\nabla T||A|^{2}\left|\boldsymbol{x}^{\top}\right| .
\end{aligned}
$$

From Theorem 3.1 we have that $|A|^{2}$ and $|H|^{2}=\left|x^{\perp}\right|^{2}$ have exponential decay, so $|A|^{2}|\boldsymbol{x}|$ and $|A|^{2}+\left|\boldsymbol{x}^{\perp}\right|^{2}$ are uniformly bounded on $L$. Thus we can find a constant $C_{2}=C_{2}(L, n, k)$ so that

$$
\left|\left\langle\Delta T, \nabla_{\boldsymbol{x} \top T} T\right\rangle-\operatorname{div}\left(\left\langle\nabla T, \nabla_{\boldsymbol{x} \top T} T\right\rangle-\boldsymbol{x}^{\top} \frac{|\nabla T|^{2}}{2}\right)\right| \leq C_{2}\left(|\nabla T|^{2}+|T|^{2}\right),
$$

which implies, after integration, inequality (32). 
Combining (31) with (32), we have

$$
\|\Delta T\|_{L^{2}}^{2}+\left\|\left\langle\boldsymbol{x}^{\top}, \nabla T\right\rangle\right\|_{L^{2}}^{2} \leq C_{3}\left(\|\phi\|_{H_{*}^{k+2}}^{2}+\|\mathcal{L}(\phi)\|_{H^{k+1}}^{2}\right)
$$

for some $C_{3}=C_{3}\left(C_{1}, C_{2}\right)$. Using the Bochner formula and the fact that $|A|^{2}$ is uniformly bounded on $L$ we can find a constant $C_{4}=C_{4}(L, n, k)$ so that

$$
\left\|\nabla^{2} T\right\|_{L^{2}}^{2} \leq\|\Delta T\|_{L^{2}}^{2}+C_{4}\|\nabla T\|_{L^{2}}^{2} .
$$

Combining this inequality with (33) we obtain

$$
\|\phi\|_{H_{*}^{k+3}}^{2} \leq C_{5}\left(\|\phi\|_{H_{*}^{k+2}}^{2}+\|\mathcal{L}(\phi)\|_{H^{k+1}}^{2}\right)
$$

for some constant $C_{5}$, which implies that $\phi \in H_{*}^{k+3}(L)$ as required.

\section{Local uniqueness}

In this section we prove the local uniqueness of zero-Maslov class Lagrangian selfexpanders $L$ asymptotic to transverse pairs of multiplicity one planes $L_{0}=P_{1}+P_{2}$.

Recall the definition of $G_{n}$ in Section 3 and consider a smooth path $\left(P_{1}(s), P_{2}(s)\right) \in G_{n}$ with $P_{1}(0)=P_{1}$ and $P_{2}(0)=P_{2}$. We assume that the difference of the Lagrangian angles $\theta\left(P_{1}(s)\right)-\theta\left(P_{2}(s)\right)$ is constant.

Theorem 5.1 Given a zero-Maslov class self-expander $L$ asymptotic to $L_{0}=P_{1}+P_{2}$, there is $R_{0}>0, s_{0}>0$ and $\varepsilon>0$ so that zero-Maslov class self-expanders $L^{s}$ that satisfy

- $L^{s}$ is asymptotic to $L_{0}^{s}=P_{1}(s)+P_{2}(s)$ for some $|s| \leq s_{0}$,

- $L^{s}$ is $\varepsilon$-close in $C^{2}$ to $L$ in $B_{R_{0}}$,

exist and are unique. The family $\left(L^{s}\right)_{|s| \leq s_{0}}$ is continuous in $C^{2, \alpha}$.

We achieve this by studying the deformation theory of $L$ and applying the Implicit Function Theorem.

We start by constructing the tubular neighbourhoods of $L_{0}$ and $L$ that we require and derive some basic properties. 


\section{Symplectic preliminaries}

The cotangent bundle of a Lagrangian $N$ has a natural symplectic structure, which is exact, meaning that there is a tautological one form $\tau \in \Lambda^{1}\left(T^{*} N\right)$ so that if $\omega_{N}$ is the tautological symplectic form on $T^{*} N$, then $\mathrm{d} \tau=-\omega_{N}$. The form $\tau$ is determined by the following property: if $\Xi \in \Lambda^{1}(N)$ and we consider the natural map $\Xi: N \rightarrow T^{*} N$, then $\Xi^{*} \tau=\Xi$. We remark that on $\mathbb{R}^{2 n}=T^{*} \mathbb{R}^{n}, \tau=\sum_{i=1}^{n} y_{i} \mathrm{~d} x_{i}$ and hence $\tau$ is different from the Liouville form $\lambda$.

For $\Xi \in \Lambda^{1}(N)$, we let $\Gamma_{\Xi}$ denote the section of $T^{*} N$ given by $x \mapsto(x, \Xi(x))$.

A symplectomorphism $\Phi:\left(M_{1}, \mathrm{~d} \lambda_{1}\right) \rightarrow\left(M_{2}, \mathrm{~d} \lambda_{2}\right)$ between exact symplectic manifolds is called exact if $\Phi^{*}\left(\lambda_{2}\right)-\lambda_{1}$ is exact.

In particular, in the case of $\mathbb{R}^{n} \subseteq \mathbb{C}^{n}$, the map from $T^{*} \mathbb{R}^{n}$ to $\mathbb{C}^{n}$ given by

$$
\left(x=\left(x_{1}, \ldots, x_{n}\right), \sum_{j=1}^{n} y_{j}(x) \mathrm{d} x_{j}\right) \mapsto\left(x_{1}+i y_{1}, \ldots, x_{n}+i y_{n}\right)
$$

is an exact symplectomorphism identifying the zero section with the real $\mathbb{R}^{n}$ in $\mathbb{C}^{n}$.

The construction of the tubular neighbourhood of $L_{0}=P_{1} \cup P_{2}$ is elementary. Without loss of generality we may assume that $P_{1}$ is the real $\mathbb{R}^{n} \subseteq \mathbb{C}^{n}$ and that $P_{2}=A \cdot \mathbb{R}^{n}$ where $A=\operatorname{diag}\left(e^{i \theta_{1}}, \ldots, e^{i \theta_{n}}\right)$. Naturally, we may define symplectomorphisms $\Psi_{j}: T^{*} P_{j} \rightarrow \mathbb{C}^{n}$ for $j=1,2$, where $\Psi_{1}$ is given by (34) and $\Psi_{2}=A \circ \Psi_{1}$. Clearly, there exists $\zeta>0$ so that if

$$
V_{j}=\left\{(x, \xi(x)) \in T^{*}\left(P_{j} \backslash\{0\}\right):|\xi(x)|<2 \zeta|x|\right\}
$$

then $\Psi_{1}\left(V_{1}\right) \cap \Psi_{2}\left(V_{2}\right)=\varnothing$. This choice ensures that we can allow for rotations of $P_{1}$ and $P_{2}$ in a tubular neighbourhood that is symplectomorphic to an open neighbourhood in $T^{*}\left(L_{0} \backslash\{0\}\right)$, so we have the following.

\section{Lemma 5.2 Set:}

- $V_{0}=V_{1} \cup V_{2} \subseteq T^{*}\left(L_{0} \backslash\{0\}\right)$.

- $T_{0}=\Psi_{1}\left(V_{1}\right) \cup \Psi_{2}\left(V_{2}\right) \subseteq \mathbb{C}^{n}$.

- $\Psi_{0}: V_{0} \rightarrow T_{0}$ defined by $\left.\Psi_{0}\right|_{V_{j}}=\Psi_{j}$.

Then $V_{0}$ and $T_{0}$ are open tubular neighbourhoods of $L_{0} \backslash\{0\}$ and $\Psi_{0}$ is a symplectomorphism preserving the Liouville form. Moreover, any small rotation of either of the planes $P_{1}$ or $P_{2}$ remains in $T_{0}$. 
We now use $V_{0}, T_{0}$ and $\Psi_{0}$ to construct our tubular neighbourhoods of $L$. The point will be to ensure that the symplectomorphism we construct is compatible with the standard symplectomorphism (34) over the planes.

By Theorem 3.1, there is $R_{1}>0$ and an open set $B \subset \mathbb{C}^{n}$ with compact closure so that

$$
L \backslash B=\left\{x+J \bar{\nabla} \psi(x): x \in L_{0} \backslash B_{R_{1}}\right\}
$$

for some $\psi \in C^{\infty}\left(L_{0} \backslash B_{R_{1}}\right)$ whose $C^{2, \alpha}$ norm decays exponentially. In particular, we may assume by making $R_{1}$ larger if necessary that $|\mathrm{d} \psi(x)|<\zeta|x|$ for all $x \in L_{0} \backslash B_{R_{1}}$. Let

$$
\pi: L_{0} \backslash B_{R_{1}} \rightarrow L \backslash B, \quad \pi(x)=x+J \bar{\nabla} \psi(x)
$$

so that $\pi^{*}: T^{*}(L \backslash B) \rightarrow T^{*}\left(L_{0} \backslash B_{R_{1}}\right)$ is an isomorphism.

Proposition 5.3 Recall the notation of Lemma 5.2. There exist

- open neighbourhoods $\widehat{V} \subset V$ of $L$ in $T^{*} L$,

- open tubular neighbourhoods $\widehat{T} \subset T$ of $L$ in $\mathbb{C}^{n}$,

- an exact symplectomorphism $\Psi: V \rightarrow T$ with $\left.\Psi\right|_{L}=\operatorname{id}_{L}$ and $\widehat{T}=\Psi(\widehat{V})$,

such that

$$
\begin{gathered}
\pi^{*}(V)=\left\{(x, \xi(x)) \in T^{*}\left(L_{0} \backslash B_{R_{1}}\right):|\xi(x)|<\zeta|x|\right\} \subseteq V_{0}, \\
\pi^{*}(\widehat{V})=\left\{(x, \xi(x)) \in T^{*}\left(L_{0} \backslash B_{R_{1}}\right):|\xi(x)|<\frac{1}{2} \zeta|x|\right\},
\end{gathered}
$$

and

$$
\Psi \circ\left(\pi^{*}\right)^{-1}(x, \xi(x))=\Psi_{0}(x, \mathrm{~d} \psi(x)+\xi(x)) \quad \text { for all }(x, \xi) \in \pi^{*}(V)
$$

Proof Recall that $L$ is embedded by Lemma 2.4.

Since $L$ is Lagrangian, the normal bundle $N L$ of $L$ is isomorphic to $T^{*} L$ so we may consider the exponential map exp acting on $T^{*} L$. Given any compact $K \subset L$, we may apply the usual tubular neighbourhood theorem to give open neighbourhoods of $K$ in $T^{*} K$ and $\mathbb{C}^{n}$ that are diffeomorphic via exp. Moreover, exp and its derivative act as the identity on $K$. In particular, $\exp ^{*} \lambda=\lambda_{L}$ on $K$, where $\lambda_{L}$ is the Liouville form on $T^{*} L$.

We may define $V$ and $\Psi$ over $L \backslash B$ via (37) and (38) so that $\Psi(V)$ is a tubular neighbourhood of $L \backslash B$. Using the exponential map over the remainder of $L$, we can extend to open neighbourhoods $V, T$ of $L$ in $T^{*} L$ and $\mathbb{C}^{n}$ and a diffeomorphism $\Phi: V \rightarrow T$ such that $\Phi$ and $\Psi$ agree over $L \backslash B$. 
As $\pi$ in (36) is a diffeomorphism, the map $\pi^{*}: T^{*}(L \backslash B) \rightarrow T^{*}\left(L_{0} \backslash B_{R_{1}}\right)$ is a symplectomorphism preserving the tautological 1-form (see, for example, Cannas da Silva [20, Theorem 2.1]). Since $\Psi_{0}$ preserves the Liouville form, we see that on $L \backslash B$ we have $\Phi^{*} \lambda-\lambda_{L}=\mathrm{d} \alpha_{L}$ for some smooth function $\alpha_{L}$. We can smoothly cut-off the function $\alpha_{L}$ so that it is defined on $T^{*} L$ and vanishes over a compact subset of $L$. Hence $\Phi$ is an exact symplectomorphism outside some compact set. The idea now is to essentially use Moser's trick to perturb $\Phi$ over a compact set to a global exact symplectomorphism.

Define

$$
\lambda_{t}=(1-t)\left(\lambda_{L}+\mathrm{d} \alpha_{L}\right)+t \Phi^{*} \lambda,
$$

so that

$$
\mathrm{d} \lambda_{t}=2(1-t) \omega_{L}+2 t \Phi^{*} \omega
$$

is a closed nondegenerate 2 -form on $V$ for all $t \in[0,1]$. Using nondegeneracy, we can uniquely solve

$$
\left.X_{t}\right\lrcorner \mathrm{d} \lambda_{t}=\lambda_{L}+\mathrm{d} \alpha_{L}-\Phi^{*} \lambda
$$

pointwise for $X_{t}$. Since $\Phi^{*} \lambda=\lambda_{L}+\mathrm{d} \alpha_{L}$ over $L \backslash B$, we see that $X_{t}$ is zero outside a compact set, so we may solve for a smooth vector field $X_{t}$ on $V$ for all $t \in[0,1]$ by shrinking $V$ if necessary. Moreover, $L$ (viewed as the zero section) is Lagrangian with respect to $\omega_{L}$ and $\Phi^{*} \omega$, so we may deduce that $\left.\mathrm{d} \lambda_{t}\right|_{L}=0$ and hence $\left.X_{t}\right|_{L}=0$ as well.

Define diffeomorphisms $f_{t}$ on $V$ such that $f_{0}=\mathrm{id}$ and $\frac{\mathrm{d}}{\mathrm{d} t} f_{t}=X_{t} \circ f_{t}$. Then

$$
\left.\left.\left.\frac{\mathrm{d}}{\mathrm{d} t} f_{t}^{*} \lambda_{t}=f_{t}^{*}\left(\Phi^{*} \lambda-\lambda_{L}-\mathrm{d} \alpha_{L}+\mathrm{d}\left(X_{t}\right\lrcorner \lambda_{t}\right)+X_{t}\right\lrcorner \mathrm{~d} \lambda_{t}\right)=\mathrm{d} f_{t}^{*}\left(X_{t}\right\lrcorner \lambda_{t}\right) .
$$

We deduce that $f_{1}^{*} \Phi^{*} \lambda-\lambda_{L}=f_{1}^{*} \lambda_{1}-f_{0}^{*} \lambda_{0}+\mathrm{d} \alpha_{L}$ is exact. Moreover the diffeomorphism $f_{1}$ acts as the identity on $L$ and on $V$ over $L \backslash B$. Hence we have an exact symplectomorphism $\Psi=\Phi \circ f_{1}: V \rightarrow T$ which satisfies (37) and (38). The remainder of the proposition follows by taking appropriate open subsets of $V$ and $T$.

Write $C_{\text {loc }}^{2}(V)$ and $C_{\text {loc }}^{2}(\hat{V})$ for the spaces of locally $C^{2}$ 1-forms $\Xi$ with graph $\Gamma_{\Xi} \subseteq V$ and $\Gamma_{\Xi} \subseteq \widehat{V}$ respectively. We use similar notation for $C_{\mathrm{loc}}^{\infty}(V)$. For $\Xi \in C_{\mathrm{loc}}^{2}(V)$ we define a $C^{2}$-embedding $f^{\Xi}: L \rightarrow T$ by

$$
f^{\Xi}(x)=\Psi(x, \Xi(x))
$$

so that $f^{\Xi}(L)$ is the deformation of $L$ given by $\Xi$.

We note that $f^{\Xi}(L)$ is Lagrangian if and only if $\mathrm{d} \Xi=0$. 
However, we want to restrict ourselves to exact zero-Maslov class deformations $f^{\Xi}(L)$ since, by Lemma 2.2, we know that if $f^{\Xi}(L)$ is a self-expander it must be exact. This motivates the next lemma.

Lemma 5.4 Let $L^{\prime}=f^{\Xi}(L)$ with $\Xi \in C_{\mathrm{loc}}^{2}(V)$. Then $L^{\prime}$ is exact and zero-Maslov class if and only if $\Xi=\mathrm{d} \phi$ for some $\phi \in C_{\mathrm{loc}}^{3}(L)$ with $\Gamma_{\mathrm{d} \phi} \subseteq V$.

Moreover, if we set

- $\bar{\phi}$ defined on $T$ so that $\bar{\phi}(\Psi(x, \xi))=\phi(x)$,

- $H\left(L^{s}\right)$ the mean curvature of $L^{s}=f^{s \mathrm{~d} \phi}(L)$ and $\Delta^{s}$ the pullback to $L$ of the Laplacian on $L^{s}$,

- $\theta^{\phi}$ the pullback to $L$ of the Lagrangian angle of $f^{\mathrm{d} \phi}(L)$, and

- $\beta^{\phi}$ the pullback to $L$ of the primitive of the Liouville form of $f^{\mathrm{d} \phi}(L)$,

then $\theta^{\phi}$ and $\beta^{\phi}$ can be given, respectively, by

$$
\theta^{\phi}(x)=\theta(x)+\int_{0}^{1} \Delta^{s} \phi(x)-\left.\left\langle H\left(L^{s}\right), \bar{\nabla} \bar{\phi}\right\rangle\right|_{f^{s \mathrm{~d} \phi}(x)} \mathrm{d} s
$$

and

$$
\beta^{\phi}(x)=\beta(x)-2 \phi(x)+\left.\int_{0}^{1}\langle x, \bar{\nabla} \bar{\phi}\rangle\right|_{f^{s \mathrm{~d} \phi}(x)} \mathrm{d} s .
$$

We also have

$$
\begin{aligned}
& \left.\frac{\mathrm{d} \theta^{s \phi}}{\mathrm{d} s}\right|_{s=0}=\Delta \phi-\langle H, \bar{\nabla} \bar{\phi}\rangle, \\
& \left.\frac{\mathrm{d} \beta^{s \phi}}{\mathrm{d} s}\right|_{s=0}=-2 \phi+\langle\boldsymbol{x}, \nabla \phi\rangle+\langle H, \bar{\nabla} \bar{\phi}\rangle .
\end{aligned}
$$

Proof We first show that exactness of $f^{\Xi}(L)$ corresponds to exactness of $\Xi$. It suffices to see that $\left(f^{\Xi}\right)^{*}\left(\sum_{i=1}^{n} y_{i} \mathrm{~d} x_{i}\right)$ is exact because that differs from $-\left(f^{\Xi}\right)^{*}(\lambda / 2)$ by an exact form. Since $\Psi$ is an exact symplectomorphism there exists a function $\alpha_{L}$ on $T^{*} L$ such that $\Psi^{*}\left(\sum_{i=1}^{n} y_{i} \mathrm{~d} x_{i}\right)=\tau+\mathrm{d} \alpha_{L}$. By definition, $f^{\Xi}=\Psi \circ \Xi$ so

$$
\left(f^{\Xi}\right)^{*}\left(\sum_{i=1}^{n} y_{i} \mathrm{~d} x_{i}\right)=\Xi^{*} \circ \Psi^{*}\left(\sum_{i=1}^{n} y_{i} \mathrm{~d} x_{i}\right)=\Xi^{*}\left(\tau+\mathrm{d} \alpha_{L}\right)=\Xi+\mathrm{d} \Xi^{*}\left(\alpha_{L}\right) \text {. }
$$

Hence $f^{\Xi}(L)$ is exact if and only if $\Xi$ is exact.

We now compute the stated identities for $\beta^{\phi}$. Consider the vector field on $T^{*} L$ given by

$$
\left.\tilde{X}\right|_{(x, \xi)}=(0, \mathrm{~d} \phi(x)) \in T_{(x, \xi)}\left(T^{*} L\right),
$$


the function $\tilde{\phi}$ on $T^{*} L$ given by $\tilde{\phi}(x, \xi)=\phi(x)$, and $X=\Psi_{*}(\tilde{X})$ a vector field on $T$. We have $\tilde{X}\lrcorner \Psi^{*} \omega=-\mathrm{d} \tilde{\phi}$ and so, because $\bar{\phi} \circ \Psi=\tilde{\phi}$, we obtain $X=J \bar{\nabla} \bar{\phi}$ on $T$. As a result we have

$$
\frac{\mathrm{d}}{\mathrm{d} s} f^{s \mathrm{~d} \phi}(x)=\left.\Psi_{*}\right|_{(x, s \mathrm{~d} \phi)}(\tilde{X})=\left.X\right|_{f} ^{s \mathrm{~d} \phi(x)}=\left.J \bar{\nabla} \bar{\phi}\right|_{f^{s \mathrm{~d} \phi}(x)} .
$$

Therefore we obtain

$$
\begin{aligned}
\frac{\mathrm{d}}{\mathrm{d} s}\left(f^{s \mathrm{~d} \phi}\right)^{*} \lambda=\left(f^{s \mathrm{~d} \phi}\right)^{*} \mathcal{L}_{J \bar{\nabla}} \bar{\phi}^{\lambda} & \left.\left.=\left(f^{s \mathrm{~d} \phi}\right)^{*}(\mathrm{~d}(J \bar{\nabla} \bar{\phi}\lrcorner \lambda)+J \bar{\nabla} \bar{\phi}\right\lrcorner 2 \omega\right) \\
& =\left(f^{s \mathrm{~d} \phi}\right)^{*} \mathrm{~d}(\langle\boldsymbol{x}, \bar{\nabla} \bar{\phi}\rangle)-2 \mathrm{~d} \phi .
\end{aligned}
$$

In light of this formula we see that if we define

$$
\beta^{\phi}(x)=\beta(x)-2 \phi(x)+\left.\int_{0}^{1}\langle\boldsymbol{x}, \bar{\nabla} \bar{\phi}\rangle\right|_{f^{s \mathrm{~d} \phi}(x)} \mathrm{d} s,
$$

we have $\left(f^{\mathrm{d} \phi}\right)^{*} \lambda=\mathrm{d} \beta^{\phi}$ and, since $L$ is a self-expander,

$$
\begin{aligned}
\left.\frac{\mathrm{d} \beta^{s \phi}}{\mathrm{d} s}\right|_{s=0}=-2 \phi+\langle\boldsymbol{x}, \bar{\nabla} \bar{\phi}\rangle & =-2 \phi+\langle\boldsymbol{x}, \nabla \phi\rangle+\left\langle\boldsymbol{x}^{\perp}, \bar{\nabla} \bar{\phi}\right\rangle \\
& =-2 \phi+\langle\boldsymbol{x}, \nabla \phi\rangle+\langle H, \bar{\nabla} \bar{\phi}\rangle
\end{aligned}
$$

We now show that the zero-Maslov class condition imposes no condition on $\Xi=\mathrm{d} \phi$. We have $\left(f^{s \mathrm{~d} \phi}\right)^{*}(\Omega)=e^{i \theta^{s}}\left(f^{s \mathrm{~d} \phi}\right)^{*} \operatorname{vol}_{L^{s}}$ where $e^{i \theta^{s}}$ is an $S^{1}$-valued function on $L$.

If the deformation vector $X$ were orthogonal to $L^{s}$, we would have by [22, Lemma 2.3] that

$$
\frac{\mathrm{d} \theta^{s}}{\mathrm{~d} s}=\Delta^{s} \phi
$$

The fact that $X$ might have a tangential component along $L^{s}$ implies

$$
\frac{\mathrm{d} \theta^{s}}{\mathrm{~d} s}=\Delta^{s} \phi+\left\langle X,\left(f^{s \mathrm{~d} \phi}\right)_{*} \nabla \theta^{s}\right\rangle=\Delta^{s} \phi+\left\langle J X, H\left(L^{s}\right)\right\rangle=\Delta^{s} \phi-\left\langle H\left(L^{s}\right), \bar{\nabla} \bar{\phi}\right\rangle .
$$

Therefore, integrating this equation for $\theta^{s}$ together with the initial condition $\theta^{0}=\theta$ allows us to define a (single-valued) function on each $L^{s}$ that is the Lagrangian angle. In particular, $f^{\mathrm{d} \phi}(L)$ has zero-Maslov class and we can set

$$
\theta^{\phi}(x)=\theta(x)+\int_{0}^{1} \Delta^{s} \phi(x)-\left.\left\langle H\left(L^{s}\right), \bar{\nabla} \bar{\phi}\right\rangle\right|_{f^{s \mathrm{~d} \phi}(x)} \mathrm{d} s .
$$

The equation for $\frac{\mathrm{d}}{\mathrm{d} s} \theta^{s \phi}$ was computed above. 


\section{Proof of Theorem 5.1}

Consider smooth rotations $P_{1}(s)$ and $P_{2}(s)$ of the planes $P_{1}$ and $P_{2}$ respectively, so that the planes remain Lagrangian and the difference of their Lagrangian angles stays the same. In that case we find a one parameter family of matrices $B_{1}(s), B_{2}(s) \in U(n)$ with

- $\quad P_{1}(s)=B_{1}(s) \cdot P_{1}$ and $P_{2}(s)=B_{2}(s) \cdot P_{2}$,

- $B_{1}(s) B_{2}^{-1}(s) \in \mathrm{SU}(n)$ and $B_{1}(0)=B_{2}(0)=\mathrm{id}$,

- $\operatorname{det} B_{1}(s)=\operatorname{det} B_{2}(s)=e^{i \theta(s)}$, where $\theta(s)$ is a smooth function with $\theta(0)=0$.

Consider $G_{s}: \mathbb{C}^{n} \rightarrow \mathbb{C}^{n}$, a one-parameter group of diffeomorphisms with $G_{0}=\mathrm{id}$,

$$
G_{s}(x)=B_{1}(s)(x) \text { on } \Psi_{1}\left(V_{1}\right) \backslash B_{R_{1}} \quad \text { and } \quad G_{s}(x)=B_{2}(s)(x) \text { on } \Psi_{2}\left(V_{2}\right) \backslash B_{R_{1}} .
$$

From Proposition 5.3 we can find $s_{0}$ so that for all $|s| \leq s_{0}$ and $\mathrm{d} \phi \in C_{\mathrm{loc}}^{3}(\hat{V})$ we have $G_{s} \circ f^{\mathrm{d} \phi}(L) \subset T$. In this case we define the $C^{2}$ embedding

$$
f^{\mathrm{d} \phi, s}: L \rightarrow T, \quad f^{\mathrm{d} \phi, s}(x)=G_{s} \circ \Psi(x, \mathrm{~d} \phi(x)) .
$$

Given $\phi \in C_{\mathrm{loc}}^{3}(L)$ so that $\mathrm{d} \phi \in C_{\mathrm{loc}}^{2}(\hat{V})$ and $|s| \leq s_{0}$, we set $L^{\phi, s}=f^{\mathrm{d} \phi, s}(L)$. Thus, by Lemma 5.4 we know that $L^{\phi, s}$ is an exact zero-Maslov class Lagrangian and we consider its Lagrangian angle $\theta^{\phi, s}$ and primitive for the Liouville form $\beta^{\phi, s}$ pulled-back to $L$, which are given by Lemma 5.4.

If $\phi$ has strong enough decay then $L^{\phi, s}$ is asymptotic to $L_{0}^{s}=P_{1}(s)+P_{2}(s)$. For simplicity we write $L^{\phi}=L^{\phi, 0}, \beta^{\phi}=\beta^{\phi, 0}$ and $\theta^{\phi}=\theta^{\phi, 0}$.

Lemma 2.2 shows that $L^{\phi, s}$ is a self-expander with $H=\boldsymbol{x}^{\perp}$ if and only if $\beta^{\phi, s}+\theta^{\phi, s}$ is constant. This motivates our definition of a deformation map.

Definition 5.5 We define a function $\widehat{F}$ on functions $\phi \in C_{\text {loc }}^{3}(L)$ such that $\mathrm{d} \phi \in$ $C_{\mathrm{loc}}^{2}(\hat{V})$ and $|s|<s_{0}$ by

$$
\widehat{F}(\phi, s)=\beta^{\phi, s}+\theta^{\phi, s}-\theta(s) .
$$

We also let $F(\phi)=\widehat{F}(\phi, 0)$.

We now compute the linearisations of $F$ and $\widehat{F}$ at zero, whose kernels will govern infinitesimal deformations of $L$. We have in fact already encountered the key operator in (11). 
Lemma 5.6 For $\phi \in C_{\mathrm{loc}}^{2}(L)$ and $s \in \mathbb{R}$,

$$
\begin{aligned}
\left.\mathrm{d} F\right|_{0}(\phi) & =\mathcal{L}(\phi)=\Delta \phi+\langle\boldsymbol{x}, \nabla \phi\rangle-2 \phi, \\
\left.\mathrm{d} \hat{F}\right|_{(0,0)}(\phi, s) & =\mathcal{L}(\phi)+s \gamma,
\end{aligned}
$$

where $\gamma$ is a smooth function with compact support.

Proof Since $\widehat{F}$ acts on a pair $(\phi, s)$, we may decompose

$$
\left.\mathrm{d} \widehat{F}\right|_{(0,0)}(\phi, s)=\left.\mathrm{d}_{1} \widehat{F}\right|_{(0,0)}(\phi)+\left.\mathrm{d}_{2} \widehat{F}\right|_{(0,0)}(s) .
$$

From Lemma 5.4 we have

$$
\left.\mathrm{d} F\right|_{0}(\phi)=\left.\frac{\mathrm{d}}{\mathrm{d} t}\left(\beta^{t \phi}+\theta^{t \phi}\right)\right|_{t=0}=\Delta \phi+\langle\boldsymbol{x}, \nabla \phi\rangle-2 \phi .
$$

Thus $\left.\mathrm{d}_{1} \widehat{F}\right|_{(0,0)}(\phi)=\mathcal{L}(\phi)$.

We note that we can find $\alpha_{s} \in C_{\mathrm{loc}}^{\infty}(L)$ so that $L^{0, s}=f^{\mathrm{d} \alpha_{s}}(L)$ and thus, using Lemma 5.4 again, we obtain

$$
\left.\mathrm{d}_{2} \widehat{F}\right|_{(0,0)}(s)=\left.\frac{\mathrm{d}}{\mathrm{d} t}\left(\beta^{0, s t}+\theta^{0, s t}\right)\right|_{t=0}-s \theta^{\prime}(0)=s\left(\mathcal{L}(\alpha)-\theta^{\prime}(0)\right)
$$

for some $\alpha \in C_{\mathrm{loc}}^{\infty}(L)$. We now argue that $\gamma=\mathcal{L}(\alpha)-\theta^{\prime}(0)$ has compact support and this finishes the proof of the lemma.

On each connected component of $T \backslash B_{R_{1}}, G_{s}$ belongs to $U(n)$ and we have $\theta^{0, s}=$ $\theta+\theta(s)$ and $\beta^{0, s}-\beta$ a constant $c(s)$ for all $|s|<s_{0}$. Next we argue that $c(s)=0$ for all $|s|<s_{0}$, which implies that

$$
\widehat{F}(0, s)=\theta^{0, s}+\beta^{0, s}-\theta(s)=\theta+\theta(s)+\beta-\theta(s)=0
$$

outside a compact set, so $\gamma$ indeed has compact support.

Recall the diffeomorphism $\pi$ given in (36). From Proposition 5.3 we see that, by choosing a larger $R_{1}$ if necessary, we can find $\chi_{s}, \psi_{s} \in C^{\infty}\left(L_{0} \backslash B_{R_{1}}\right)$ with $\chi_{0}=0$, $\psi_{0}=\psi$, respectively, so that, for all $x \in L_{0} \backslash B_{R_{1}}$ and $|s| \leq s_{0}$, we have

$$
f^{0, s}(\pi(x))=G_{s} \circ \Psi_{0}(x, \mathrm{~d} \psi(x))=\Psi_{0}\left(x, \mathrm{~d} \chi_{s}+\mathrm{d} \psi_{s}(x)\right) .
$$

Note that $\chi_{s}$ is a homogeneous quadratic polynomial and the $C^{2}$ norm of $\psi_{s}$ decays exponentially. Therefore we have from Lemma 5.4 that

$$
c(s)=\beta^{0, s}(\pi(x))-\beta(\pi(x))=-2 \chi_{s}+\left\langle x, \nabla \chi_{s}\right\rangle+o\left(|x|^{-1}\right) .
$$

Since $\chi_{s}$ is a homogeneous quadratic, we have $-2 \chi_{s}+\left\langle x, \nabla \chi_{s}\right\rangle=0$ and thus $c(s)=0$ for all $|s|<s_{0}$. 
We show that the nonlinear map $F$ is well-defined for $\phi$ in some open ball about zero in $H_{*}^{k+2}(L)$ for all $k$ large.

Proposition 5.7 If $k>1+\frac{n}{2}$, there exists $\varepsilon_{0}>0$ so that

$$
F: B_{\varepsilon_{0}}(0) \subset H_{*}^{k+2}(L) \rightarrow H^{k}(L) \quad \text { and } \quad \widehat{F}: B_{\varepsilon_{0}}(0) \times\left(-s_{0}, s_{0}\right) \rightarrow H^{k}(L)
$$

are well-defined.

Proof If $k>1+\frac{n}{2}$ then the Sobolev Embedding Theorem implies that $H^{k+2} \hookrightarrow C^{3, \alpha}$ for some $\alpha \in(0,1)$ with $\alpha<k-1-\frac{n}{2}$, so there is an open set containing zero in $H^{k+2}$ on which $F$ is defined. The existence of $\varepsilon_{0}$ so that $F(\phi)$ and $\widehat{F}(\phi, s)$ are defined for $\phi \in B_{\varepsilon_{0}}(0)$ and $|s|<s_{0}$ is then immediate as $H_{*}^{k+2} \subset H^{k+2}$.

We need to show that $F$ and $\hat{F}$ take $B_{\varepsilon_{0}}(0)$ into $H^{k}(L)$. Note that the conditions satisfied by $G_{s} \in U(n)$ in $T \backslash B_{R_{1}}$ imply that, outside $B_{R_{1}}, \theta^{\phi, s}=\theta^{\phi}+\theta(s)$ and $\beta^{\phi, s}$ differs from $\beta^{\phi}$ by a constant $c(\phi, s)$. Since $\phi$ tends to zero at infinity in $C^{3}$, we have that $c(s, \phi)=c(s)$, and we saw in the proof of Lemma 5.6 that $c(s)=0$. Thus $\beta^{\phi, s}$ is identical to $\beta^{\phi}$ and so $\widehat{F}(\phi, s)$ and $F(\phi)$ are identical functions outside $B_{R_{1}}$. Hence, we only need to argue that $F$ takes $B_{\varepsilon_{0}}(0)$ into $H^{k}(L)$.

Notice that $\theta^{\phi}$ depends only on the tangent space of $L^{\phi}$, and thus on $\nabla \phi$ and $\nabla^{2} \phi$. Thus we can consider a smooth function of its arguments $Q_{\theta}(x, y, z)$ so that

$$
\theta^{\phi}(x)=\theta(x)+\Delta \phi(x)-\langle H, \bar{\nabla} \bar{\phi}\rangle+Q_{\theta}\left(x, \nabla \phi(x), \nabla^{2} \phi(x)\right) .
$$

Using the expression for the linearisation of $\theta^{\phi}$ given in Lemma 5.4 and arguing as in Joyce [9, Proposition 2.10], we conclude that $Q_{\theta}, \partial_{y} Q_{\theta}$ and $\partial_{z} Q_{\theta}$ vanish at $(x, 0,0)$.

From the expression for $\beta^{\phi}$ given in Lemma 5.4 we see that we can find a smooth function of its arguments $Q_{\beta}(x, y)$ so that

$$
\beta^{\phi}(x)=\beta(x)+\langle\boldsymbol{x}, \nabla \phi(x)\rangle-2 \phi(x)+\langle H, \bar{\nabla} \bar{\phi}\rangle+Q_{\beta}(x, \nabla \phi(x)) .
$$

Using the expression for the linearisation of $\beta^{\phi}$ given in Lemma 5.4 and again arguing as in [9, Proposition 2.10], we conclude that $Q_{\beta}$ and $\partial_{y} Q_{\beta}$ vanish at $(x, 0)$.

Therefore, since $F(0)=\theta+\beta=0$, we see that

$$
F(\phi)(x)=\mathcal{L}(\phi)(x)+Q\left(x, \nabla \phi(x), \nabla^{2} \phi(x)\right),
$$

where $Q=Q(x, y, z)$ is a smooth function of its arguments such that $Q, \partial_{y} Q$ and $\partial_{z} Q$ all vanish at $(x, 0,0)$. Observe that $Q(x, y, z)$ does not directly depend on $\phi(x)$. By Proposition 4.2 it is now enough to show that $Q$ takes $B_{\varepsilon_{0}}(0)$ into $H^{k}(L)$. 
Let $\phi \in B_{\varepsilon_{0}}(0)$ be a smooth function with compact support. We derive estimates for $\eta$ given by $\eta(x)=Q\left(x, \nabla \phi(x), \nabla^{2} \phi(x)\right)$. Since $Q$ and its first derivatives in $y$ and $z$ vanish when $\phi=0$,

$$
|\eta(x)| \leq C(x)\left(|\nabla \phi(x)|^{2}+\left|\nabla^{2} \phi(x)\right|^{2}\right)
$$

for some non-negative function $C$ on $L$. Our first objective is to show that $C$ is bounded.

Recall we have a diffeomorphism $\pi: L_{0} \backslash B_{R_{1}} \rightarrow L \backslash B$ and set $\phi_{0}=\pi^{*}(\phi), F_{0}(\phi)=$ $\pi^{*}(F(\phi))$. From Proposition 5.3 we have

$$
f^{\phi, 0}(\pi(x))=\Psi_{0}\left(x, \mathrm{~d} \psi(x)+\mathrm{d} \phi_{0}(x)\right)
$$

and so on $L_{0} \backslash B_{R_{1}}$ we have $F_{0}(\phi)=\theta_{0}^{\phi}+\beta_{0}^{\phi}$, where $\theta_{0}^{\phi}$ and $\beta_{0}^{\phi}$ are the pull backs of the Lagrangian angle and primitive for $\lambda$ on the graph of $\mathrm{d} \phi_{0}+\mathrm{d} \psi$ over $L_{0} \backslash B_{R_{1}}$. Thus,

$$
F_{0}(\phi)(x)=\mathcal{L}_{0}\left(\phi_{0}+\psi\right)(x)+Q_{0}\left(x, \nabla\left(\phi_{0}+\psi\right)(x), \nabla^{2}\left(\phi_{0}+\psi\right)(x)\right),
$$

where $\mathcal{L}_{0}$ is the operator given in (11) calculated on $L_{0}$ and $Q_{0}$ is a function with the same properties as $Q$. Since we are working over the planes $L_{0}$, we compute

$$
\beta_{0}^{\phi}=\beta_{0}+\left\langle\boldsymbol{x}, \nabla\left(\phi_{0}+\psi\right)\right\rangle-2\left(\phi_{0}+\psi\right) \quad \text { and } \quad \theta_{0}^{\phi}=\theta_{0}+\sum_{j=1}^{n} \tan ^{-1}\left(\mu_{j}\right)
$$

where $\mu_{1}, \ldots, \mu_{n}$ are the eigenvalues of Hess $\left(\phi_{0}+\psi\right)$, and $\theta_{0}, \beta_{0}$ are the Lagrangian angle and primitive for the Liouville form on $L_{0}$. Thus, because we have chosen $\theta_{0}+\beta_{0}=0$, we have

$$
Q_{0}\left(x, \nabla\left(\phi_{0}+\psi\right)(x), \nabla^{2}\left(\phi_{0}+\psi\right)(x)\right)=\sum_{j=1}^{n} \tan ^{-1}\left(\mu_{j}\right)(x)-\Delta\left(\phi_{0}+\psi\right)(x) .
$$

From this explicit formula, we deduce that $Q_{0}$ and all its derivatives are bounded on $L_{0} \backslash B_{R_{1}}$. Moreover, the decay of $\left|\left(\partial_{x}\right)^{a} Q_{0}(x, y, z)\right|$ is controlled by $|y|^{2}+|z|^{2}$. The exponential decay of $\psi$ in Theorem 3.1 implies $Q$ and $Q_{0}$ differ by terms with exponential decay and so we have that $Q$ and all its derivatives are bounded on $L$, and that the $x$ derivatives of $Q$ satisfy

$$
\left|\left(\partial_{x}\right)^{a} Q\left(x, \nabla \phi(x), \nabla^{2} \phi(x)\right)\right| \leq C^{a}\left(|\nabla \phi(x)|^{2}+\left|\nabla^{2} \phi(x)\right|^{2}\right)
$$

for some constants $C^{a}$. In particular, we can choose $C(x)=C$ independent of $x$ in (39) and we deduce that

$$
\|\eta\|_{L^{2}} \leq C\|\phi\|_{C^{2}}\|\phi\|_{H^{2}} .
$$


Since any element of $H_{*}^{k+2}$ has bounded $C^{2}$ norm and lies in $H^{2}$, we deduce that $Q$ maps $B_{\varepsilon_{0}}(0)$ into $L^{2}$.

Now let $j \in\{1, \ldots, k\}$. Our aim is to show that $\nabla^{j} \eta$ lies in $L^{2}$. By the chain rule,

$$
\begin{aligned}
\left|\nabla^{j} \eta\right| \leq j ! \sum_{\substack{a, b, c \geq 0 \\
a+b+c \leq j}}\left|\left(\partial_{x}\right)^{a}\left(\partial_{y}\right)^{b}\left(\partial_{z}\right)^{c} Q\right| & \\
\times \sum_{\substack{m_{1}, \ldots, m_{b}, n_{1}, \ldots, n_{c} \geq 1 \\
a+m_{1}+\cdots+m_{b}+n_{1}+\cdots+n_{c}=j}} & \prod_{i=1}^{b}\left|\nabla^{m_{i}}(\nabla \phi)\right| \prod_{l=1}^{c}\left|\nabla^{n_{l}}\left(\nabla^{2} \phi\right)\right| .
\end{aligned}
$$

If $j=a$ in the sum in (41), the products are trivial and we can use (40) to show that the corresponding terms lie in $L^{2}$. Therefore we now assume that $j>a$. Let $q_{1}, \ldots, q_{b}, r_{1}, \ldots, r_{c}$ be positive constants so that

$$
\sum_{i=1}^{b} \frac{1}{q_{i}}+\sum_{l=1}^{c} \frac{1}{r_{l}}=1 .
$$

Applying Hölder's inequality to (41), we see that

$$
\begin{aligned}
\int_{L}\left|\nabla^{j} \eta\right|^{2} \mathrm{~d} \mathcal{H}^{n} & \sum_{\substack{a, b, c \geq 0 \\
a+b+c \leq j}} C(a, b, c) \prod_{\substack{m_{1}, \ldots, m_{b}, n_{1}, \ldots, n_{c} \geq 1 \\
a+m_{1}+\cdots+m_{b}+n_{1}+\cdots+n_{c}=j}} \prod_{i=1}^{b}\left(\int_{L}\left|\nabla^{m_{i}}(\nabla \phi)\right|^{2 q_{i}} \mathrm{~d} \mathcal{H}^{n}\right)^{1 / q_{i}} \\
& \times \prod_{l=1}^{c}\left(\int_{L}\left|\nabla^{n_{l}}\left(\nabla^{2} \phi\right)\right|^{2 r_{l}} \mathrm{~d} \mathcal{H}^{n}\right)^{1 / r_{l}}
\end{aligned}
$$

for some constants $C(a, b, c)$ determined by $j$ and the derivatives of $Q$, which are bounded.

Given a section $\sigma$ of a vector bundle with a connection $D$ over $L$ that lies in $H^{s} \cap L^{\infty}$ we have by Cantor [2, Theorem 3] that

$$
\int_{L}\left|D^{p} \sigma\right|^{2 s / p} \mathrm{~d} \mathcal{H}^{n} \leq C\|\sigma\|_{\infty}^{2(s / p)-2}\|\sigma\|_{H^{s}}^{2}
$$

for some constant $C$ independent of $\sigma$, whenever $s \geq p$. (Notice that the results in [2] apply since $L$ is complete, has injectivity radius bounded away from zero and bounded sectional curvature.) Choosing $q_{i}=(j-a) / m_{i}$ and $r_{l}=(j-a) / n_{l}$, we see that (42) holds and we can apply (44) to deduce that there exists some constant $C$, independent 
of $\phi$, so that

$$
\begin{aligned}
& \int_{L}\left|\nabla^{m_{i}}(\nabla \phi)\right|^{2 q_{i}} \mathrm{~d} \mathcal{H}^{n} \leq C\|\nabla \phi\|_{\infty}^{2 q_{i}-2}\|\phi\|_{H^{j-a+1}}^{2}, \\
& \int_{L}\left|\nabla^{n_{l}}\left(\nabla^{2} \phi\right)\right|^{2 r_{l}} \mathrm{~d} \mathcal{H}^{n} \leq C\left\|\nabla^{2} \phi\right\|_{\infty}^{2 r_{l}-2}\|\phi\|_{H^{j-a+2}}^{2} .
\end{aligned}
$$

Therefore, substituting (45) and (46) into (43) we see that there exists a constant $C\left(j, Q,\|\phi\|_{C^{2}}\right)$ so that

$$
\int_{L}\left|\nabla^{j} \eta\right|^{2} \mathrm{~d} \mathcal{H}^{n} \leq C\left(j, Q,\|\phi\|_{C^{2}}\right)\|\phi\|_{H^{j+2}}^{2} .
$$

Since this holds for all smooth compactly supported $\phi \in B_{\varepsilon}(0)$ we see that $\eta \in H^{j}$ whenever $\phi \in H^{j+2} \cap C^{2}$ for $j=1, \ldots, k$. The result for $F$ follows.

We can now prove the following local uniqueness result.

Theorem 5.8 Let $k>1+\frac{n}{2}$. There exist $0<\varepsilon_{1} \leq \varepsilon_{0}$ and $0<s_{1} \leq s_{0}$ so that for each $|s|<s_{1}$ there exists a unique $\phi(s) \in B_{\varepsilon_{1}}(0) \subseteq H_{*}^{k+2}(L)$ so that $\widehat{F}(\phi(s), s)=0$, where $s \mapsto \phi(s)$ is continuous.

Moreover, if $(\phi, s) \in B_{\varepsilon_{1}}(0) \times\left(-s_{1}, s_{1}\right)$ then $L^{\phi, s}$ is a self-expander with $H=x^{\perp}$ if and only if $\phi=\phi(s)$.

Proof By Proposition 5.7, $\widehat{F}: B_{\varepsilon_{0}}(0) \times\left(-s_{0}, s_{0}\right) \rightarrow H^{k}(L)$ is well-defined and $L^{\phi, s}$ is a self-expander if and only if $\widehat{F}(\phi, s)$ is constant. However, if $\phi \in B_{\varepsilon_{0}}(0)$ then $\widehat{F}(\phi, s) \in C^{1}(L) \cap L^{2}(L)$ by the Sobolev Embedding Theorem and so $|\widehat{F}(\phi, s)(x)| \rightarrow 0$ as $|x| \rightarrow \infty$. Hence $L^{\phi, s}$ is a self-expander if and only if $\widehat{F}(\phi, s)=0$.

By Lemma 5.6, d $\left.\widehat{F}\right|_{(0,0)}(\phi, s)=\mathcal{L}(\phi)+s \gamma$ and $\gamma$ has compact support. Corollary 4.5 implies that $\left.\mathrm{d}_{1} \widehat{F}\right|_{(0,0)}=\mathcal{L}: H_{*}^{k+2}(L) \rightarrow H^{k}(L)$ is an isomorphism. Thus

$$
\left.\mathrm{d} \widehat{F}\right|_{(0,0)}: H_{*}^{k+2}(L) \times \mathbb{R} \rightarrow H^{k}(L)
$$

is surjective. Moreover, there exists unique $\Gamma \in H_{*}^{k+2}(L)$ such that $\mathcal{L}(\Gamma)=-\gamma$, so $\left.\mathrm{d} \widehat{F}\right|_{(0,0)}$ has a $1-$ dimensional kernel.

Applying the Implicit Function Theorem for Banach spaces (Lang [12, Chapter XIV, Theorem 2.1]), we see that there exist $\varepsilon_{1} \leq \varepsilon_{0}, s_{1} \leq s_{0}$ and a unique continuous map $s \mapsto \phi(s)$ so that

$$
\widehat{F}^{-1}(0) \cap\left(B_{\varepsilon_{1}}(0) \times\left(-s_{1}, s_{1}\right)\right)=\left\{(\phi(s), s):|s|<s_{1}\right\} .
$$

The result follows. 
We now finish the proof of Theorem 5.1.

From Theorem 5.8 we obtain, for all $|s|<s_{1}$, the existence of a zero-Maslov class selfexpander $L^{s}$ asymptotic to $L_{0}^{s}=P_{1}(s)+P_{2}(s)$. The family $\left(L_{S}\right)_{|s|<s_{1}}$ is continuous in $C^{2, \alpha}$.

To show uniqueness, apply Theorem 3.1 with $k=3$ and

$$
K=\left\{\left(P_{1}(s), P_{2}(s)\right)\right\}_{|s| \leq s_{1}} \subset G_{n}
$$

to obtain the existence of $\varepsilon$ and $R_{0}$ so that if $N^{s}$ is a self-expander asymptotic to $L_{0}^{s}$ that is $\varepsilon$-close in $C^{2}$ to $L$ in $B_{R_{0}}$, then $N^{s}=L^{\phi, s}$ for some $\phi \in B_{\varepsilon_{1}}$. Theorem 5.8 implies $N^{s}$ is unique and equal to $L^{s}$.

\section{Compactness theorem in $\mathbb{C}^{2}$}

We now restrict to the situation where the self-expander is asymptotic to transverse planes in $\mathbb{C}^{2}$. The reason is that it is only in $\mathbb{C}^{2}$ where a Lagrangian cone with density strictly less than 2 must be a plane. For $n>2$, the Harvey-Lawson $U(1)^{n-1}$-invariant special Lagrangian cone in $\mathbb{C}^{n}$ has density strictly between 1 and 2.

Consider $M=G_{L}\left(2, \mathbb{C}^{2}\right) \times G_{L}\left(2, \mathbb{C}^{2}\right)$, where $G_{L}\left(2, \mathbb{C}^{2}\right)$ denotes the set of all multiplicity one Lagrangian planes in $\mathbb{C}^{2}$. Define

$$
S L=\left\{\left(P_{1}, P_{2}\right) \in M \mid P_{1}+P_{2} \text { or } P_{1}-P_{2} \text { is area-minimizing }\right\}
$$

and

$$
\Lambda=\left\{\left(P_{1}, P_{2}\right) \in M \mid P_{1} \cap P_{2}=\{0\}\right\} \backslash S L .
$$

Since $\left(P_{1}, P_{2}\right) \in M$ lies in $S L$ if and only if the sum of the angles between $P_{1}$ and $P_{2}$ is an integer multiple of $\pi$, we see that $\Lambda$ is an open subset of $M$.

The aim of this section is to prove the following compactness result.

Theorem 6.1 Pick a compact set $K \subset \Lambda$. The set

$\mathcal{S}(K)=\left\{L \subseteq \mathbb{C}^{2} \mid L\right.$ is a zero-Maslov class Lagrangian self-expander that is asymptotic to $P_{1}+P_{2}$, where $\left(P_{1}, P_{2}\right) \in K$ \}

is compact in the $C^{2, \alpha}$ topology.

Proof Let $\left(L^{i}\right)_{i \in \mathbb{N}}$ be a sequence of self-expanders in $\mathcal{S}(K)$ asymptotic to $L_{0}^{i}=$ $P_{1}^{i}+P_{2}^{i}$. Setting $L_{t}^{i}=\sqrt{2 t} L^{i}$, we thus have a sequence $\left(L_{t}^{i}\right)_{t \geq 0}$ of solutions to 
Lagrangian mean curvature flow that are smooth for all $t>0$. From Lemma 2.4 we have uniform area bounds for $\left(L_{t}^{i}\right)_{t \geq 0}$ and so Ilmanen [8, Theorem 7.1] implies that we can consider a subsequence that converges weakly to an integral Brakke motion $\left(L_{t}\right)_{t \geq 0}$.

It also follows from [8, Theorem 7.1] that, for almost all $t>0, L_{t}^{i}$ admits a subsequence that converges to $L_{t}$ as an integral varifold and so $2 t H=x^{\perp}$ on $L_{t}$. Furthermore, Radon measure convergence implies that $L_{t}=\sqrt{2 t} L_{1 / 2}$ for all $t>0$. In particular, $L_{1 / 2}$ is an integral varifold with $H=x^{\perp}$ and we denote it simply by $L$.

Compactness of $K$ implies that, after passing to another subsequence, $\left(P_{1}^{i}, P_{2}^{i}\right)$ converges to $\left(P_{1}, P_{2}\right) \in K$. Our objective is to show that $L \in \mathcal{S}(K)$.

We first show that $L$ is asymptotic to $L_{0}=P_{1}+P_{2}$.

Lemma 6.2 There is $R_{0}>0$ and $\psi \in C^{\infty}\left(L_{0} \backslash B_{R_{0}}\right)$ so that

$$
L \backslash B_{2 R_{0}} \subset\left\{x+J \bar{\nabla} \psi(x) \mid x \in L_{0} \backslash B_{R_{0}}\right\} \subset L \backslash B_{R_{0} / 2}
$$

and, for some $b>0$,

$$
\|\psi\|_{C^{3, \alpha}\left(L_{0} \backslash B_{R}\right)}=O\left(e^{-b R^{2}}\right) \quad \text { as } R \rightarrow \infty .
$$

Moreover, $L^{i} \backslash B_{R_{0}}$ converges to $L \backslash B_{R_{0}}$ in $C^{2, \alpha}$ as $i \rightarrow \infty$.

Proof The lemma follows from Theorem 3.1 applied to $\left(L^{i}\right)_{i \in \mathbb{N}}$.

We can now deduce that $L$ is asymptotic to $L_{0}$.

Lemma 6.3 As Radon measures, $L_{t} \rightarrow L_{0}$ as $t \rightarrow 0$.

Proof Given $\varepsilon>0$ small we obtain from Lemma 6.2 that, for all $t$ sufficiently small,

$$
L_{t} \backslash B_{2 \varepsilon} \subset\left\{x+J Y_{t}(x) \mid x \in L_{0} \backslash B_{\varepsilon}\right\} \subset L_{t} \backslash B_{\varepsilon / 2},
$$

where the $C^{2, \alpha}$ norm of the vector field $Y_{t}$ tends to zero as $t \rightarrow 0$. Thus,

$$
\lim _{t \rightarrow 0^{+}} \int_{L_{t}} \phi \mathrm{d} \mathcal{H}^{2}=\int_{L_{0}} \phi \mathrm{d} \mathcal{H}^{2}
$$

for all $\phi \in C_{0}^{\infty}\left(\mathbb{C}^{2}\right)$.

The next proposition is one of the key steps to ensure that $L$ is smooth.

Proposition 6.4 $L$ is not a stationary varifold. 
Proof Assume $L$ is stationary. Then $L$ needs to be a cone because $\boldsymbol{x}^{\perp}=H=0$. Thus $L_{t}=\sqrt{2 t} L$ has $H=0$ for all $t>0$ and we obtain from Lemma 6.3 that $L=P_{1}+P_{2}$. The goal for the rest of this proof is to show that $L$ must be area-minimizing and this gives us a contradiction because $\left(P_{1}, P_{2}\right) \notin S L$.

Since $L^{i}$ is a self-expander we have (from varifold convergence) that for every $r>0$

$$
\lim _{i \rightarrow \infty} \int_{L^{i} \cap B_{r}}\left|x^{\perp}\right|^{2} \mathrm{~d} \mathcal{H}^{2}=\int_{L \cap B_{r}}\left|x^{\perp}\right|^{2} \mathrm{~d} \mathcal{H}^{2}=0
$$

and thus, for all $r>0$,

$$
\lim _{i \rightarrow \infty} \int_{L^{i} \cap B_{r}}\left(|H|^{2}+\left|x^{\perp}\right|^{2}\right) \mathrm{d} \mathcal{H}^{2}=\lim _{i \rightarrow \infty} \int_{L^{i} \cap B_{r}}\left(2\left|x^{\perp}\right|^{2}\right) \mathrm{d} \mathcal{H}^{2}=0 .
$$

Lemma 6.5 The following properties hold:

(i) There is $d_{0}>0$ so that for every $R>0$, every $i$ sufficiently large, and every open subset $A$ of $L^{i} \cap B_{4 R}$ with rectifiable boundary we have

$$
\left(\mathcal{H}^{2}(A)\right)^{\frac{1}{2}} \leq d_{0} \mathcal{H}^{1}(\partial A) .
$$

(ii) There is $R_{1}>0$ so that for all $R>R_{1}$ and all $i$ sufficiently large

$$
L^{i} \cap B_{2 R} \text { is connected and } \partial\left(L^{i} \cap B_{3 R}\right) \subset \partial B_{3 R} .
$$

(iii) There is $c>0$ so that for all $i$ sufficiently large we have

$$
\sup _{L^{i}}\left|\theta^{i}\right|=\sup _{L^{i}}\left|\beta^{i}\right| \leq c .
$$

Proof We first prove (i). From the Michael-Simon Sobolev inequality (see Simon [21, Theorem 18.6])

$$
\left(\mathcal{H}^{2}(A)\right)^{\frac{1}{2}} \leq c_{0} \int_{A}|H|+c_{0} \mathcal{H}^{1}(\partial A)
$$

for some universal constant $c_{0}>0$. In this case we have

$$
\left(\mathcal{H}^{2}(A)\right)^{\frac{1}{2}} \leq c_{0}\left(\mathcal{H}^{2}(A)\right)^{1 / 2}\left(\int_{A}|H|^{2}\right)^{\frac{1}{2}}+c_{0} \mathcal{H}^{1}(\partial A)
$$

and so we get the desired claim because for all $i$ sufficiently large we have (due to (48))

$$
c_{0}^{2} \int_{L^{i \cap B_{4 R}}}|H|^{2} \leq \frac{1}{4} .
$$

Property (ii) follows from Lemma 6.2. 
Finally, we prove property (iii). Given $y_{i} \in L^{i}$, denote by $\widehat{B}_{r}\left(y_{i}\right)$ the intrinsic ball in $L^{i}$ of radius $r$ and set $\psi_{i}(r)=\mathcal{H}^{2}\left(\widehat{B}_{r}\left(y_{i}\right)\right)$. From (i) we see that for almost all $r$

$$
\left(\psi_{i}(r)\right)^{\frac{1}{2}} \leq d_{0} \mathcal{H}^{1}\left(\partial \widehat{B}_{r}\left(y_{i}\right)\right)=d_{0} \psi_{i}^{\prime}(r) .
$$

Integrating the above inequality implies the existence of $d_{1}>0$, depending only on $d_{0}$, so that for all $R>0$

$$
\mathcal{H}^{2}\left(\widehat{B}_{r}\left(y_{i}\right)\right) \geq d_{1} r^{2} \quad \text { for all } y_{i} \in B_{3 R} \cap L^{i} \text { and } r<R .
$$

Choose $\beta^{i}$, the primitive for the Liouville form $\left.\lambda\right|_{L_{i}}$, so that $\beta^{i}+\theta^{i}=0$ ( $L^{i}$ is a self-expander). Combining the uniform area bounds given in Lemma 2.4 with (49), we have that the intrinsic diameter of $L^{i} \cap B_{R}$ is uniformly bounded for all $i$ sufficiently large. Hence, if $x, y \in L^{i} \cap B_{R}$ and $\gamma$ is a path in $L^{i} \cap B_{R}$ connecting $x$ to $y$, we have

$$
\beta^{i}(x)-\beta^{i}(y)=\int_{\gamma} \lambda \leq R \text { length }(\gamma) .
$$

Thus the oscillation of $\beta^{i}$ in $L^{i} \cap B_{R}$ is uniformly bounded. The angle $\theta^{i}=-\beta^{i}$ can always be chosen so that its range in $L^{i} \cap B_{R}$ intersects the interval $[0,2 \pi]$ and so we obtain that $\theta^{i}=-\beta^{i}$ is uniformly bounded in $B_{R}$.

From Lemma 6.2 we know that $\theta^{i}=-\beta^{i}$ are uniformly bounded outside a large ball and thus are uniformly bounded on $L^{i}$.

We can now finish the proof of Proposition 6.4. Recall that $L=P_{1}+P_{2}$ in the varifold sense and, if necessary, we can change the orientation of one of the planes so that the identity also holds in the current sense. We want to show that $L=P_{1}+P_{2}$ is area-minimizing.

We know that for all $R>0$,

$$
\lim _{i \rightarrow \infty} \int_{L^{i} \cap B_{R}}|H|^{2}+\left|x^{\perp}\right|^{2} \mathrm{~d} \mathcal{H}^{2}=0
$$

and $\left|\boldsymbol{x}^{\perp}\right|=\left|\nabla \beta^{i}\right|$ is uniformly bounded in $B_{R}$. From Lemma 6.5 we have that all conditions necessary to apply Neves' [16, Proposition A.1] are met and so we conclude the existence of a constant $\bar{\beta}$ and $R_{2}$ such that, for all $\phi \in C_{0}^{\infty}\left(\mathbb{C}^{2}\right)$,

$$
\lim _{i \rightarrow \infty} \int_{L^{i} \cap B_{R_{2}}}\left(\beta^{i}-\bar{\beta}\right)^{2} \phi \mathrm{d} \mathcal{H}^{2}=0 .
$$

Hence

$$
\lim _{i \rightarrow \infty} \int_{L^{i} \cap B_{R_{2}}}\left(\theta^{i}+\bar{\beta}\right)^{2} \phi \mathrm{d} \mathcal{H}^{2}=0
$$


for all $\phi \in C_{0}^{\infty}\left(\mathbb{C}^{2}\right)$. We deduce, from [16, Proposition 5.1], that $L$ has constant Lagrangian angle $-\bar{\beta}$ and is thus area-minimizing, providing our required contradiction.

Using the fact that $L$ is not stationary, we now show that $L$ satisfies the conditions of White's Regularity Theorem. It is in this lemma that we use the fact that $n=2$ in a crucial way. Recall the definition of Gaussian density in (3).

Lemma 6.6 Given $\varepsilon_{0}>0$ small, there is $\delta>0$ so that

$$
\Theta_{t}(y, l) \leq 1+\frac{\varepsilon_{0}}{2} \quad \text { for every } l \leq \delta t, y \in \mathbb{C}^{2} \text { and } t>0 .
$$

Remark 6.7 We briefly sketch the idea. The first step is to find $\delta$ so that $\Theta_{1 / 2}(y, l)<2$ for all $y \in \mathbb{C}^{n}$ and $l \leq \delta$. This follows because the monotonicity formula implies that $\Theta_{1 / 2}(y, l) \leq \Theta_{0}\left(y, l+\frac{1}{2}\right) \leq 2$ with equality only if $L$ is a self-shrinker centered at the origin. In the latter case, because $L$ is a self-expander, we obtain that $L$ must be stationary, which contradicts Proposition 6.4. Thus, the strict inequality holds as claimed.

The second step is to show that if $\Theta_{1 / 2}\left(y_{i}, \delta_{i}\right) \geq 1+\frac{\varepsilon_{0}}{2}$ for some sequence $\delta_{i}$ tending to zero, then we can blow-up $L$ and obtain a stationary Lagrangian varifold $\tilde{L}$ that is not a plane. Then we blow-down $\widetilde{L}$ to obtain a stationary Lagrangian cone $C$ that must have Gaussian density at the origin bigger than $1+\frac{\varepsilon_{0}}{2}$. Since we are in $\mathbb{C}^{2}$, this forces the Gaussian density at the origin to be at least two, which we then show contradicts the first step.

Proof It suffices to prove the lemma for $t=\frac{1}{2}$ because $L_{t}=\sqrt{2 t} L$.

In what follows we will constantly use the fact that, because $P_{1}$ intersects $P_{2}$ transversely,

$$
\int_{P_{1}+P_{2}} \Phi(y, l) \mathrm{d} \mathcal{H}^{2}<2 \text { for all } l>0 \text { and } y \neq 0,
$$

with equality holding if $y=0$.

First step We start by arguing the existence of $c_{1}>0$ such that, for every $l \leq 2$ and $y \in \mathbb{C}^{2}$,

$$
\int_{L} \Phi(y, l) \mathrm{d} \mathcal{H}^{2} \leq 2-c_{1}^{-1}
$$


From the monotonicity formula for Brakke flows [7, Lemma 7],

$$
\begin{aligned}
\int_{L} \Phi(y, l) \mathrm{d} \mathcal{H}^{2}+\int_{0}^{\frac{1}{2}} \int_{L_{t}}\left|H+\frac{(x-y)^{\perp}}{2\left(l+\frac{1}{2}-t\right)}\right|^{2} \Phi\left(y, l+\frac{1}{2}-t\right) \mathrm{d} \mathcal{H}^{2} \mathrm{~d} t \\
=\int_{P_{1}+P_{2}} \Phi\left(y, l+\frac{1}{2}\right) \mathrm{d} \mathcal{H}^{2} \leq 2 .
\end{aligned}
$$

Suppose there is a sequence $y_{i}$ and $l_{i}$ with $0 \leq l_{i} \leq 2$ such that

$$
\int_{L} \Phi\left(y_{i}, l_{i}\right) \mathrm{d} \mathcal{H}^{2} \geq 2-\frac{1}{i} .
$$

Then, by (51),

$$
\int_{P_{1}+P_{2}} \Phi\left(y_{i}, l_{i}+\frac{1}{2}\right) \mathrm{d} \mathcal{H}^{2} \geq 2-\frac{1}{i}
$$

and so $y_{i}$ must converge to zero.

Assuming without loss of generality that $l_{i}$ converges to $\bar{l}$, we have again from (51) that

$$
\begin{gathered}
\int_{0}^{\frac{1}{2}} \int_{L_{t}}\left|H+\frac{x^{\perp}}{2\left(\bar{l}+\frac{1}{2}-t\right)}\right|^{2} \Phi\left(0, \bar{l}+\frac{1}{2}-t\right) \mathrm{d} \mathcal{H}^{2} \mathrm{~d} t \\
=\lim _{i \rightarrow \infty} \int_{0}^{\frac{1}{2}} \int_{L_{t}}\left|H+\frac{\left(x-y_{i}\right)^{\perp}}{2\left(l_{i}+\frac{1}{2}-t\right)}\right|^{2} \Phi\left(y_{i}, l_{i}+\frac{1}{2}-t\right) \mathrm{d} \mathcal{H}^{2} \mathrm{~d} t \\
\leq 2-\lim _{i \rightarrow \infty} \int_{L} \Phi\left(y_{i}, l_{i}\right) \mathrm{d} \mathcal{H}^{2}=0
\end{gathered}
$$

and thus

$$
H+\frac{x^{\perp}}{2\left(\bar{l}+\frac{1}{2}-t\right)}=0 \quad \text { on } L_{t} \text { for almost all } t \in\left[0, \frac{1}{2}\right] .
$$

Combining this with the fact that $H=x^{\perp} /(2 t)$ on $L_{t}$ we obtain that $L$ must be stationary, which contradicts Proposition 6.4. Thus (50) must hold.

Second step To finish the proof we argue again by contradiction and assume the existence of sequences $\left(y_{j}\right)_{j \in \mathbb{N}}$ in $\mathbb{C}^{2}$ and $\left(\delta_{j}\right)_{j \in \mathbb{N}}$ converging to zero so that

$$
\Theta_{\frac{1}{2}}\left(y_{j}, \delta_{j}\right) \geq 1+\frac{\varepsilon_{0}}{2} .
$$

From the monotonicity formula for Brakke flows [7, Lemma 7] we have

$$
\int_{P_{1}+P_{2}} \Phi\left(y_{j}, \delta_{j}+\frac{1}{2}\right) \mathrm{d} \mathcal{H}^{2} \geq \Theta_{1 / 2}\left(y_{j}, \delta_{j}\right) \geq 1+\frac{\varepsilon_{0}}{2} .
$$


Note that the sequence $\left(\left|y_{j}\right|\right)_{j \in \mathbb{N}}$ is bounded by a positive constant $M_{0}$, because otherwise we could find a subsequence so that

$$
\lim _{j \rightarrow \infty} \int_{P_{1}+P_{2}} \Phi\left(y_{j}, \delta_{j}+\frac{1}{2}\right) \leq 1 .
$$

Consider the sequence of blow-ups

$$
\widetilde{L}_{s}^{j, i}=\delta_{j}^{-1 / 2}\left(L_{1 / 2+s \delta_{j}}^{i}-y_{j}\right) \quad \text { with } s \geq 0 .
$$

A standard diagonalization argument allows us to consider a subsequence $\widetilde{L}_{s}^{j}=\widetilde{L}_{s}^{j, i(j)}$ such that, for all $1 \leq l \leq j$,

$$
-\frac{1}{j} \leq \int_{\tilde{L}_{0}^{j}} \Phi(0, l) \mathrm{d} \mathcal{H}^{2}-\int_{L} \Phi\left(y_{j}, l \delta_{j}\right) \mathrm{d} \mathcal{H}^{2} \leq \frac{1}{j} .
$$

Thus, for every $r>0$,

$$
\begin{aligned}
\int_{0}^{1} \int_{\tilde{L}_{s}^{j} \cap B_{r}}|H|^{2} \mathrm{~d} \mathcal{H}^{2} \mathrm{~d} s & =\delta_{j}^{-1} \int_{1 / 2}^{1 / 2+\delta_{j}} \int_{L_{t}^{i(j)} \cap B_{\sqrt{\delta}_{j} r}\left(y_{j}\right)}|H|^{2} \mathrm{~d} \mathcal{H}^{2} \mathrm{~d} t \\
& =\delta_{j}^{-1} \int_{1 / 2}^{1 / 2+\delta_{j}} \int_{L_{t}^{i(j)} \cap B_{\sqrt{\delta}_{j} r}\left(y_{j}\right)}\left|\frac{x^{\perp}}{2 t}\right|^{2} \mathrm{~d} \mathcal{H}^{2} \mathrm{~d} t \leq c_{2} \delta_{j},
\end{aligned}
$$

where $c_{2}$ depends on $r$ and $M_{0}$. Therefore

$$
\lim _{j \rightarrow \infty} \int_{0}^{1} \int_{\widetilde{L}_{s}^{j} \cap B_{r}}|H|^{2} \mathrm{~d} \mathcal{H}^{2} \mathrm{~d} s=0
$$

and so $\left(\tilde{L}_{\mathcal{S}}^{j}\right)_{0 \leq s \leq 1}$ converges to an integral Brakke flow $\left(\tilde{L}_{s}\right)_{0 \leq s \leq 1}$ with $\widetilde{L}_{s}=\tilde{L}$ for all $s$ and $\widetilde{L}$ a stationary varifold. From [16, Proposition 5.1] we conclude that $\widetilde{L}$ is a union of special Lagrangian currents (the Lagrangian angle is uniformly bounded by Lemma 6.5(iii)).

From (52) and (53) we have

$$
\int_{\widetilde{L}} \Phi(0,1) \mathrm{d} \mathcal{H}^{2} \geq 1+\frac{\varepsilon_{0}}{2}
$$

and so $\tilde{L}$ cannot be a plane with multiplicity one. The blow-down

$$
C=\lim _{i \rightarrow 0} \varepsilon_{i} \widetilde{L}, \quad \text { where } \varepsilon_{i} \rightarrow 0,
$$


is a union of Lagrangian planes (as these are the only special Lagrangian cones in $\mathbb{C}^{2}$ ) and so

$$
\lim _{i \rightarrow \infty} \int_{\widetilde{L}} \Phi\left(0, \varepsilon_{i}^{-2}\right) \mathrm{d} \mathcal{H}^{2}=\lim _{i \rightarrow \infty} \int_{\varepsilon_{i} \tilde{L}} \Phi(0,1) \mathrm{d} \mathcal{H}^{2}=\int_{C} \Phi(0,1) \mathrm{d} \mathcal{H}^{2} \geq 2 .
$$

From (53) this implies that one can find $l$ such that for every $j$ sufficiently large we have

$$
2-\frac{1}{2 c_{1}} \leq \int_{\tilde{L}_{0}^{j}} \Phi(0, l) \mathrm{d} \mathcal{H}^{2} \leq \int_{L} \Phi\left(y_{j}, l \delta_{j}\right) \mathrm{d} \mathcal{H}^{2}+\frac{1}{j} .
$$

This contradicts (50).

We may now complete the proof of Theorem 6.1. From Lemmas 6.2 and 6.6 we have that, for all $i$ sufficiently large,

$$
\Theta_{t}^{i}(y, l) \leq 1+\varepsilon_{0} \quad \text { for every } l \leq \delta t, y \in \mathbb{C}^{2} \text { and } t>0,
$$

where $\Theta_{t}^{i}(y, l)$ is the Gaussian density (3) of $L_{t}^{i}$.

White's Regularity Theorem [23] implies uniform $C^{2, \alpha}$ bounds for $L_{1 / 2}^{i}$ and so $L=L_{1 / 2}$ is a smooth multiplicity one self-expander asymptotic to $P_{1}+P_{2}$ with $\left(P_{1}, P_{2}\right) \in K$ and $L_{1 / 2}^{i}$ converges to $L$ in $C^{2, \alpha}$. Hence, $L \in \mathcal{S}(K)$ as we wanted to show.

\section{Uniqueness theorem in $\mathbb{C}^{2}$}

We first prove the uniqueness for self-expanders that are asymptotic to planes $P_{1}+P_{2}$, where $P_{1}$ and $P_{2}$ share the same $S^{1}$-symmetry.

\section{Equivariant case}

We say a Lagrangian surface $N \subset \mathbb{C}^{2}$ is equivariant if there is a curve $\gamma: \mathbb{R} \rightarrow \mathbb{C}$ or $\gamma:[0, \infty) \rightarrow \mathbb{C}$ so that

$$
N=\{(\gamma(s) \cos \alpha, \gamma(s) \sin \alpha) \mid s \in \mathbb{R}, \alpha \in[0,2 \pi]\} \subset \mathbb{C}^{2} .
$$

Consider the ambient function $\mu=x_{1} y_{2}-y_{2} x_{1}$. The relevance of this function is that an embedded Lagrangian $N$ is equivariant if and only if $N \subset \mu^{-1}(0)$ (see Neves [18, Lemma 7.1] for instance).

Studying the o.d.e. arising from $H=\boldsymbol{x}^{\perp}$, Anciaux [1] showed that given two equivariant planes $P_{1}, P_{2}$, there is a unique equivariant Lagrangian self-expander $L$ asymptotic to $L_{0}=P_{1}+P_{2}$. 
Lemma 7.1 Suppose that $L$ is a zero-Maslov class Lagrangian self-expander asymptotic to $L_{0}=P_{1}+P_{2}$, where $P_{1}, P_{2}$ are equivariant planes. Then $L$ is equivariant. In particular, it is unique.

Proof From [17, Lemma 3.3] we know that along $L_{t}=\sqrt{2 t} L$,

$$
\frac{\mathrm{d}}{\mathrm{d} t} \mu^{2}=\Delta \mu^{2}-2|\nabla \mu|^{2} \text {. }
$$

Using the evolution equation above in Huisken's monotonicity formula we have that, for $t<1$,

$$
\frac{\mathrm{d}}{\mathrm{d} t} \int_{L_{t}} \mu^{2} \Phi(0,1-t) \mathrm{d} \mathcal{H}^{2} \leq 0
$$

From Theorem 3.1 we see that

$$
\lim _{t \rightarrow 0} \int_{L_{t}} \mu^{2} \Phi(0,1-t) \mathrm{d} \mathcal{H}^{2}=\int_{P_{1}+P_{2}} \mu^{2} \Phi(0,1) \mathrm{d} \mathcal{H}^{2}=0
$$

because $P_{1}, P_{2}$ are equivariant. Thus

$$
\int_{L_{t}} \mu^{2} \Phi(0,1-t) \mathrm{d} \mathcal{H}^{2}=0
$$

for all $t<1$ and this implies the desired result.

\section{General case}

Consider the set $\Lambda \subset G_{L}\left(2, \mathbb{C}^{2}\right) \times G_{L}\left(2, \mathbb{C}^{2}\right)$ defined in (47):

$$
\Lambda=\left\{\left(P_{1}, P_{2}\right) \mid P_{1} \cap P_{2}=\{0\}\right\} \backslash S L .
$$

Theorem 7.2 Given $\left(P_{1}, P_{2}\right) \in \Lambda$ there is a unique zero-Maslov class self-expander $L$ that is asymptotic to $L_{0}=P_{1}+P_{2}$.

Remark 7.3 The existence of such self-expanders was proven by Joyce, Lee and Tsui in [10]. Explicit formulae for the self-expanders are given in [10, Theorem C].

Proof Choose a basis of $\mathbb{C}^{2}$ so that $P_{1}$ is the real plane and

$$
P_{2}=\left\{\left(e^{i \theta_{1}} x, e^{i \theta_{2}} y\right) \mid x, y \in \mathbb{R}\right\} .
$$

Set

$$
P_{2}(s)=\left\{\left(e^{i \theta_{1}-i s\left(\theta_{1}-\theta_{2}\right) / 2} x, e^{i \theta_{2}+i s\left(\theta_{1}-\theta_{2}\right) / 2} y\right) \mid x, y \in \mathbb{R}\right\}
$$


The key properties of $\left(P_{1}, P_{2}(s)\right)$ are that

- $\left(P_{1}, P_{2}(s)\right) \in \Lambda$ for all $0 \leq s \leq 1$ and the Lagrangian angle of $P_{2}(s)$ is constant,

- $P_{1} \subset \mu^{-1}(0)$ and $P_{2}(1) \subset \mu^{-1}(0)$.

Consider the compact subset of $\Lambda$ given by $K=\left\{\left(P_{1}, P_{2}(s)\right)\right\}_{0 \leq s \leq 1}$ and recall $\mathcal{S}(K)=\left\{L \subseteq \mathbb{C}^{2} \mid L\right.$ is a zero-Maslov class Lagrangian self-expander that is asymptotic to $P_{1}+P_{2}$, where $\left.\left(P_{1}, P_{2}\right) \in K\right\}$.

Consider the obvious projection map $\pi: \mathcal{S}(K) \rightarrow[0,1]$.

From [10, Theorem C] we know that $\pi$ is surjective. By Theorem 5.1, one may choose a suitable topology on $\mathcal{S}(K)$ so that $\pi$ is a local diffeomorphism. By Theorem 6.1, $\mathcal{S}(K)$ is also compact with respect to this topology. Therefore $\pi$ is a covering map. However, by Lemma 7.1, we have that $\pi^{-1}$ (1) consists of a single element and so $\pi$ is a diffeomorphism. In particular, $\pi^{-1}(0)$ consists of a single element.

\section{References}

[1] H Anciaux, Construction of Lagrangian self-similar solutions to the mean curvature flow in $\mathbb{C}^{n}$, Geom. Dedicata 120 (2006) 37-48 MR2252892

[2] M Cantor, Sobolev inequalities for Riemannian bundles, from: "Differential geometry, Part 2", (S S Chern, R Osserman, editors), Amer. Math. Soc. (1975) 171-184 MR0380873

[3] I Castro, A M Lerma, Hamiltonian stationary self-similar solutions for Lagrangian mean curvature flow in the complex Euclidean plane, Proc. Amer. Math. Soc. 138 (2010) 1821-1832 MR2587467

[4] A Chau, J Chen, W He, Entire self-similar solutions to Lagrangian mean curvature flow arXiv:0905.3869

[5] K Ecker, Regularity theory for mean curvature flow, Progress in Nonlinear Differential Equations and their Applications 57, Birkhäuser, Boston, MA (2004) MR2024995

[6] G Huisken, Asymptotic behavior for singularities of the mean curvature flow, J. Differential Geom. 31 (1990) 285-299 MR1030675

[7] T Ilmanen, Singularities of mean curvature flow of surfaces Available at http:// www. math.ethz.ch/ ilmanen/papers/sing.ps

[8] T Ilmanen, Elliptic regularization and partial regularity for motion by mean curvature, Mem. Amer. Math. Soc. 108 (1994) 90 MR1196160

[9] D Joyce, Special Lagrangian submanifolds with isolated conical singularities, II: Moduli spaces, Ann. Global Anal. Geom. 25 (2004) 301-352 MR2054572 
[10] D Joyce, Y-I Lee, M-P Tsui, Self-similar solutions and translating solitons for Lagrangian mean curvature flow, J. Differential Geom. 84 (2010) 127-161 MR2629511

[11] N V Krylov, Lectures on elliptic and parabolic equations in Hölder spaces, Graduate Studies in Mathematics 12, Amer. Math. Soc. (1996) MR1406091

[12] S Lang, Real and functional analysis, 3rd edition, Graduate Texts in Mathematics 142, Springer, New York (1993) MR1216137

[13] Y-I Lee, M-T Wang, Hamiltonian stationary shrinkers and expanders for Lagrangian mean curvature flows, J. Differential Geom. 83 (2009) 27-42 MR2545029

[14] Y-I Lee, M-T Wang, Hamiltonian stationary cones and self-similar solutions in higher dimension, Trans. Amer. Math. Soc. 362 (2010) 1491-1503 MR2563738

[15] H Nakahara, Some examples of self-similar solutions and translating solitons for mean curvature flow arXiv:1109.3554

[16] A Neves, Singularities of Lagrangian mean curvature flow: Zero-Maslov class case, Invent. Math. 168 (2007) 449-484 MR2299559

[17] A Neves, Recent progress on singularities of Lagrangian mean curvature flow, from: "Surveys in geometric analysis and relativity", (H L Bray, W P Minicozzi II, editors), Adv. Lect. Math. 20, International Press (2011) 413-438 MR2906935

[18] A Neves, Finite time singularities for Lagrangian mean curvature flow, Ann. of Math. 177 (2013) 1029-1076 MR3034293

[19] A Neves, G Tian, Translating solutions to Lagrangian mean curvature flow, Trans. Amer. Math. Soc. 365 (2013) 5655-5680

[20] A Cannas da Silva, Lectures on symplectic geometry, Lecture Notes in Mathematics 1764, Springer, Berlin (2001) MR1853077

[21] L Simon, Lectures on geometric measure theory, Proc. Centre Math. Analysis 3, Australian Nat. Univ. Centre Math. Analysis, Canberra (1983) MR756417

[22] R P Thomas, S-T Yau, Special Lagrangians, stable bundles and mean curvature flow, Comm. Anal. Geom. 10 (2002) 1075-1113 MR1957663

[23] B White, A local regularity theorem for mean curvature flow, Ann. of Math. 161 (2005) 1487-1519 MR2180405

Department of Mathematics, University College London

Gower Street, London WC1E 6BT, UK

Imperial College London

Huxley Building, 180 Queen's Gate, London SW7 2RH, UK

j.lotay@ucl.ac.uk, a.neves@imperial.ac.uk

Proposed: Tobias H Colding

Seconded: Richard Thomas, Leonid Polterovich

Received: 14 August 2012

Accepted: 29 April 2013 